\title{
Effective algebraic integration in bounded genus
}

\author{
Jorge Vitório Pereira and Roberto Svaldi
}

\begin{abstract}
We introduce and study birational invariants for foliations on projective surfaces built from the adjoint linear series of positive powers of the canonical bundle of the foliation. We apply the results to investigate the effective algebraic integration of foliations on the projective plane. In particular, we describe the Zariski closure of the set $\Sigma_{d, g}$ of foliations on $\mathbb{P}^{2}$ of degree $d$ admitting rational first integrals with fibers having geometric genus bounded by $g$.
\end{abstract}

\section{Introduction}

\subsection{Effective algebraic integration}

It seems fair to say that the simplest class of algebraic ordinary differential equations consists of the class of equations having all their solutions algebraic. In general, given an explicit differential equation, it is a difficult problem to decide whether or not it belongs to this distinguished class. Perhaps the first positive result on the subject is Schwarz's list of parameters for which Gauss' hypergeometric equation has an algebraic solution [Sch73].

Motivated by this remarkable result, a lot of activity on the study of algebraic solutions of linear differential equations took place in the nineteenth century, leading to a fairly good understanding of the problem for homogeneous linear differential equations. Among the works dealing with this question, one can find contributions by Fuchs, Gordan, Jordan, Halphen, and Klein, just to name a few. At that time, the community seemed to believe that it would be possible to decide whether or not all solutions of a given linear differential equation are algebraic. For instance, Klein concludes [Kle56, Chapter V, Section 3] with the remark that "Thus is the problem, which we formulated at the beginning of this paragraph [present all linear homogeneous differential equations of the second order with rational coefficients: $y^{\prime \prime}+p y^{\prime}+q y=0$ which possess altogether algebraic solutions], fully solved."

By the end of the nineteenth century, mathematicians like Painlevé, Autonne, and Poincaré [Poi91, Poi97] started to study the next case, that is, polynomial differential equations of first order and of first degree. In modern language, they studied foliations on the projective plane with special emphasis on the existence of methods/algorithms to decide whether or not all leaves

Received 27 April 2017, accepted in final form 3 May 2018.

2010 Mathematics Subject Classification 37F75, $14 \mathrm{E} 99$.

Keywords: holomorphic foliations, effective algebraic integration, degree of invariant algebraic curves.

This journal is (c) Foundation Compositio Mathematica 2019. This article is distributed with Open Access under the terms of the Creative Commons Attribution Non-Commercial License, which permits non-commercial reuse, distribution, and reproduction in any medium, provided that the original work is properly cited. For commercial re-use, please contact the Foundation Compositio Mathematica.

The first author is partially supported by Cnpq and FAPERJ. The second author was partially supported by NSF research grants no: 1200656 and no: 1265263. During the final revision of this work, he was supported by funding from the European Union's Seventh Framework Programme (FP7/2007-2013)/ERC Grant agreement no. 307119 . 


\section{EFFECTIVE ALGEBRAIC INTEGRATION IN BOUNDED GENUS}

are algebraic. We will call this general line of inquiry effective algebraic integration. The results obtained at that time relied on strong assumptions on the nature of the singularities of the foliations and were not considered definitive, as one can learn from the introduction ${ }^{1}$ of [Poi97]. For a modern account of some of these classical results, see [GZ97] and [Per03, Chapter 7].

The results of the nineteenth century on the effective integration of linear differential equations were revisited in the course of the twentieth century. It was then made clear that a full solution for the problem was not available; rather, the problem was reduced to a similar one for rank one linear differential equations over curves. More precisely, in order to be able to decide whether or not a homogeneous linear differential equation $P\left(x, y, y^{\prime}, y^{\prime \prime}, y^{\prime \prime \prime}, \ldots, y^{(n)}\right)=0$ has all its solutions algebraic, it suffices to be able to solve the following problem: given an element $u$ belonging to an algebraic extension of the field $\mathbb{C}(x)$, decide whether $u$ is the logarithmic derivative of an element $v$ also belonging to an algebraic extension of $\mathbb{C}(x)$. Some authors expressed doubts about the possibility of solving this problem. For instance, in [Har71, Chapter V, Section 19(i), p. 51], one can find the view of $\mathrm{Hardy}^{2}$ on the subject.

Despite the skepticism of Hardy and others (cf. [Ris70]), in the late 1960s, Risch (ibid) showed that this problem, in turn, can be reduced to the following one: given an explicit divisor on an explicit algebraic curve $C$, decide whether or not the divisor is of finite order in the Jacobian of $C$. Risch proved that this problem can be solved by restricting the data modulo two distinct primes and using the resulting bounds in positive characteristic to devise an explicit bound in characteristic zero. For a detailed account of the case of second-order homogeneous differential equations; see [BD79]. The interested reader can find more about the history of effective algebraic integration of linear differential equations in [vdPS03, Section 4.3.1, p. 124], [Gra08, Chapter III], and the references therein.

The corresponding problem for (non-linear) differential equations of the first order and of the first degree is still wide open and received considerably less attention. After the problem was dormant for a good while, the interest towards it has been revived by experts in foliation theory who considered the problem of bounding the degree of algebraic leaves of foliations on $\mathbb{P}^{2}$; see for instance [CL91, Car94, CC97, EK03] and references therein. The influence of arithmetic on the subject was rediscovered by Lins Neto [Lin02], who determined algebraic families (pencils) of foliations on the projective plane with fixed number and analytical type of singularities and with algebraic leaves of arbitrarily large degree.

\subsection{Degenerations of planar foliations admitting a rational first integral}

This work investigates the problem of effective algebraic integration for foliations on projective surfaces. In order to focus the discussion and clarify the framework in which we are going to carry it, we introduce the following conjecture.

Conjecture 1.1. The Zariski closure in $\mathbb{P} H^{0}\left(\mathbb{P}^{2}, T_{\mathbb{P}^{2}}(d-1)\right)$ of the set of foliations of degree $d$ on $\mathbb{P}^{2}$ which admit a rational integral consists of transversely projective foliations.

\footnotetext{
1 "Je me suis occupé de nouveau de la même question dans ces derniers temps, dans l' espoir que je parviendrais à généraliser les résultats obtenus. Cet espoir a été déçu. J'ai obtenu cependant quelques résultats partiels, que je prends la liberté de publier, estimant qu'on pourra s'en servir plus tard pour obtenir, par un nouvel effort, une solution plus satisfaisante du problème."

2 "But no method has been devised as yet by which we can always determine in a finite number of steps whether a given elliptic integral is pseudo-elliptic, and integrate it if it is, and there is reason to suppose that no such method can be given."
} 


\section{J. V. Pereira And R. Svaldi}

This conjecture is inspired by a remark made by Painlevé ${ }^{3}$ [Pai97, Douzième Leçon, Conclusions, pp. 216bis-217] in his Stockholm lectures. Knowledge of a transversely projective structure for a given foliation, in view of their recent description [CP14, LPT16], would allow one to reduce the problem to either the determination of periods of differential forms - when, after passing to a ramified covering, the foliation is defined by a closed rational 1-form - or to the algebraic integrability of Riccati equations.

The main results of this paper provide evidence in favor of this conjecture and are obtained using birational techniques. More precisely, we use basic results on adjoint linear series, the birational classification of foliated surfaces according to their Kodaira dimension [McQ08, Bru04, Men00], to obtain a variant of the classification which we now proceed to explain.

\subsection{Adjoint dimension of foliations}

The works of the Italian school of algebraic geometry in the beginning of the twentieth century showed how much of the geometry of a smooth projective surface $X$ can be determined by the order of growth of the function

$$
n \mapsto h^{0}\left(X, K_{X}^{\otimes n}\right) .
$$

Whenever this function grows slower than a quadratic polynomial, one has a rather precise description of the surface (the so-called Enriques-Kodaira classification). A similar classification is also available in dimension three thanks to the works of the modern school of birational geometry, and there is also a similar picture in arbitrary dimensions conditional on the so-called abundance conjecture.

In the case of foliations on surfaces, McQuillan, Brunella, and Mendes obtained a very precise classification, analogous to the Enriques-Kodaira classification, in terms of the Kodaira dimension of the foliation. As in the case of surfaces, the Kodaira dimension $\operatorname{kod}(\mathcal{F})$ of a foliation $\mathcal{F}$ measures the growth of the function $h^{0}\left(X, K_{\mathcal{F}}{ }^{\otimes n}\right)$, where $K_{\mathcal{F}}$ is the bundle of holomorphic 1 -forms along the leaves of the foliation.

As the terminology suggests, the canonical bundle together with its dual is the most obvious naturally determined line bundle on a variety. Combined with the fact that the integers $h^{0}\left(X, K_{X}^{\otimes n}\right)(n>0)$ are birational invariants for smooth projective varieties, its study is rather natural if one wants to understand varieties birationally. For foliations of arbitrary dimension or codimension, besides the canonical bundle, one also has another naturally attached line bundle: the determinant of the conormal bundle. If $\mathcal{F}$ is a foliation on a projective surface $X$ with canonical singularities, then it turns out that for arbitrary $n, m \geqslant 0$, the integers $h^{0}\left(X, K_{\mathcal{F}}{ }^{\otimes n} \otimes N_{\mathcal{F}}^{*} \otimes m\right)$ are birational invariants. Most of the results obtained in this paper stem from this simple observation. We define the adjoint dimension of a foliation according to the order of growth of the function $h^{0}\left(X, K_{\mathcal{F}}^{\otimes n} \otimes N_{\mathcal{F}}^{*}{ }^{\otimes m}\right)$; see Section 3 .

Building on the classification of foliations on surfaces according to their Kodaira dimension, in Section 6 we present a classification in function of the adjoint dimension. The results we obtain are summarized in Table 1. The outcome of the classification provides a framework well-suited to deal with families of foliations (Section 7), mainly due to the fact that it is more flexible with respect to the type of singularities which are allowed (Section 4). The classification in terms of the adjoint

\footnotetext{
3 “J'ajoute qu'on ne peut espérer résoudre d'un coup qui consiste à limiter $n$. L'énoncé vers lequel il faut tendre doit avoir la forme suivante: 'On sait reconnaître si l'intégrale dune équation $F(y /, y, x)=0$ donnée est algébrique ou ramener l'équation aux quadratures.' Dans ce dernier cas, la question reviendrait à reconnaître si une certaine intégrale abélienne (de première ou de troisième espèce) n'a que deux ou une périodes."
} 


\section{EFFECTIVE ALGEBRAIC INTEGRATION IN BOUNDED GENUS}

dimension also reflects distinct cases of the problem of effective algebraic integration (Section 8).

\begin{tabular}{|l|l|l|}
\hline adj & kod & Description \\
\hline \hline$-\infty$ & $-\infty$ & Rational fibration \\
\cline { 2 - 3 } & 0 & Finite quotient of Riccati foliation generated by global vector field \\
\cline { 2 - 3 } & 1 & Riccati foliation \\
\hline \hline 0 & 0 & Finite quotient of linear foliation on a torus \\
\hline \hline \multirow{2}{*}{1} & 0 & Finite quotient of $E \times C \rightarrow C, g(C) \geqslant 2$ \\
\cline { 2 - 3 } & 1 & Finite quotient of $E \times C \rightarrow E, g(C) \geqslant 2$ \\
\cline { 2 - 3 } & 1 & Turbulent foliation \\
\cline { 2 - 3 } & 1 & Non-isotrivial elliptic fibration \\
\hline \hline \multirow{2}{*}{2} & $-\infty$ & Irreducible quotient of $\mathbb{H} \times \mathbb{H} \rightarrow \mathbb{H}$ \\
\cline { 2 - 3 } & 1 & Finite quotient of $C_{1} \times C_{2} \rightarrow C_{1}, g\left(C_{i}\right) \geqslant 2$ \\
\cline { 2 - 3 } & 2 & General type \\
\hline
\end{tabular}

TABLE 1. Classification of foliations according to their adjoint and Kodaira dimensions

\subsection{Plan of the paper and statement of the main results}

The bulk of the paper starts by reviewing the classification of foliations with respect to their Kodaira dimension, in Section 2. Then we introduce new birational invariants for foliations on surfaces, notably the effective threshold and the adjoint dimension, in Section 3. Section 4 is devoted to the study of a variation of the concept of canonical singularities, the so-called $\varepsilon$ canonical singularities. We prove in Corollary 4.10 that for $\varepsilon>0$, this concept is stable for small perturbations of the singularity of the foliation. This fact will be particularly important in the study of families of foliations carried out in Section 7.

Section 5 is devoted to the proof of the boundedness of non-isotrivial fibrations of bounded genus in families; see Theorem 5.4. In the particular case of $\mathbb{P}^{2}$, the result reads as follows.

Theorem A. Let $\mathcal{F}$ be a foliation on $\mathbb{P}^{2}$. Assume that $\mathcal{F}$ is birationally equivalent to a nonisotrivial fibration of genus $g \geqslant 2$. Then the degree of the general leaf of $\mathcal{F}$ is bounded by

$$
(4(42(2 g-2)) !)^{2}(4 g-4) \operatorname{deg}(\mathcal{F}) .
$$

Theorem A refines the main result of [Per02], which establishes the existence of a bound for the degree of the general leaf depending on its genus and on the first $k>0$ for which the linear system $\left|K_{\mathcal{F}}{ }^{\otimes k}\right|$ defines a rational map with two-dimensional image. The existence of a universal $k$ working for every non-isotrivial fibration of genus $g$ was not known then and is still not known at the present time; hence, the existence of a bound depending only on the degree of the foliation and on the genus was unclear. In comparison to [Per02], the proof of the result above has two new ingredients. The first is a bound on the multiplicities of irreducible components of fibers of relatively minimal non-isotrivial fibrations of genus $g \geqslant 2$; cf. Proposition 5.3. The second new ingredient is the use of standard results on adjoint linear series (recalled in Section 5.1) in order to obtain effective $(n, m) \in \mathbb{N}^{2}$ such that the rational map defined by $\left|K_{\mathcal{F}}{ }^{\otimes n} \otimes K_{X}{ }^{\otimes m}\right|$ has a two-dimensional image. By imposing further assumptions on the nature of the singularities of a foliation on $\mathbb{P}^{2}$, we obtain significantly better bounds (sub-linear on $g$ ), refining a classical result of Poincaré; cf. Theorem 5.6. 


\section{J. V. Pereira And R. Svaldi}

In Section 6, we carry out the classification of foliations on surfaces according to the adjoint dimension; see Table 1. The proof strongly relies on the classification of foliations according to the Kodaira dimension, but we do need to carefully deal with its subtlest point: the classification of non-abundant foliations. A nice corollary of the classification is a cohomological characterization of rational fibrations, which is a weak analogue of Castelnuovo's criterion for the rationality of surfaces; cf. [Bea96, Theorem V.1].

THEOREM B. Let $\mathcal{F}$ be a foliation with at worst canonical singularities on a smooth projective

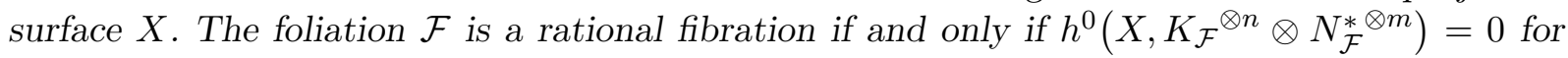
every $n \geqslant 1$ and every $m>0$.

Section 7 investigates families of foliations. There, it is shown that the set of effective thresholds in a family does not accumulate at zero (Theorem 7.6). More importantly, it prepares the ground for the proof of the most compelling evidence we have so far in favor of Conjecture 1.1.

Theorem C. The Zariski closure in $\mathbb{P} H^{0}\left(\mathbb{P}^{2}, T_{\mathbb{P}^{2}}(d-1)\right)$ of the set of degree $d$ foliations admitting a rational first integral with general fiber of genus at most $g$ is formed by transversely projective foliations.

This theorem's proof is presented in Section 8 and relies on Theorem A, on the birational classification of foliations, and on basic properties of families of foliations.

\section{Kodaira dimension of foliations}

We start things off by reviewing the birational classification of foliations on surfaces following [McQ08] and [Bru04]. No new results are presented in this section. We have only included proofs of a few key properties of the Zariski decomposition of the canonical bundle of a foliation which will be used further on.

\subsection{Singularities of foliations}

Definition 2.1. Let $\mathcal{F}$ be a foliation on $X$, and let $\pi: Y \rightarrow X$ be a birational morphism. Denote the pull-back of $\mathcal{F}$ under $\pi$ by $\mathcal{G}$. If $E$ is an exceptional divisor of $\pi$, then the discrepancy of $\mathcal{F}$ along $E$ is

$$
a(\mathcal{F}, E)=\operatorname{ord}_{E}\left(K_{\mathcal{G}}-\pi^{*} K_{\mathcal{F}}\right) .
$$

Definition 2.2. Let $\mathcal{F}$ be a foliation on $X$. A point $x \in X$ is canonical for $\mathcal{F}$ if and only if $a(\mathcal{F}, E) \geqslant 0$ for every divisor $E$ over $x$. A point $x \in X$ is $\log$ canonical for $\mathcal{F}$ if and only $a(\mathcal{F}, E) \geqslant-1$ for every divisor $E$ over $x$.

Example 2.3. Consider the pencil of foliations on $X=\mathbb{P}^{2}$ defined by the vector fields $s x \frac{\partial}{\partial x}+t y \frac{\partial}{\partial y}$, where $(s: t) \in \mathbb{P}^{1}$. If $s \cdot t \cdot(s-t) \neq 0$, then $\mathcal{F}_{(s: t)}$ is a foliation with trivial canonical bundle and three singularities at the points $(0: 0: 1),(0: 1: 0)$, and $(1: 0: 0)$. For $(s: t) \notin \mathbb{P}^{1}(\mathbb{Q})$, the three singularities are canonical. For $(s: t) \in \mathbb{P}^{1}(\mathbb{Q})-\{(0: 1),(1: 0),(1: 1)\}$, two of the singularities are log canonical but not canonical, while the third singularity is canonical. Finally, when $s \cdot t \cdot(s-t)=0$, the vector field will have one of the coordinate axes as a line of singularities. The corresponding foliation will have canonical bundle $\mathcal{O}_{\mathbb{P}^{2}}(-1)$ and only one singularity, which is $\log$ canonical but not canonical.

Any foliation on a projective surface is birationally equivalent to a foliation having at worst canonical singularities thanks to the following, which is essentially due to Seidenberg. 


\section{EFFECTIVE ALGEBRAIC INTEGRATION IN BOUNDED GENUS}

TheOREM 2.4. Let $\mathcal{F}$ be a foliation on a smooth projective surface $X$. Then there exists a finite composition of blow-ups $\pi: Y \rightarrow X$ such that all the singularities of $\pi^{*} \mathcal{F}$ are canonical.

\subsection{Kodaira dimension}

Definition 2.5. Let $\mathcal{F}$ be a foliation with at worst canonical singularities on a smooth projective surface $X$. The Kodaira dimension $\operatorname{kod}(\mathcal{F})$ of $\mathcal{F}$ is by definition

$$
\operatorname{kod}(\mathcal{F}):=\operatorname{kod}\left(K_{\mathcal{F}}\right)=\max _{m \in \mathbb{N}}\left\{\operatorname{dim} \phi_{m}(X)\right\},
$$

where $\phi_{m}$ is the rational map from $X$ to $\mathbb{P}\left(H^{0}\left(X, K_{\mathcal{F}}{ }^{\otimes m}\right)^{*}\right)$ induced by the linear system $\left|H^{0}\left(X, K_{\mathcal{F}}^{\otimes m}\right)\right|$, and we adopt the convention that $\operatorname{dim} \phi_{m}(X)=-\infty$ when $h^{0}\left(X, K_{\mathcal{F}}{ }^{\otimes m}\right)=0$ (and it is not possible to define the associated map).

The numerical Kodaira dimension $\nu(\mathcal{F})$ of $\mathcal{F}$ is defined to be the numerical dimension of $K_{\mathcal{F}}$, that is,

$-\nu(\mathcal{F})=-\infty$ if $K_{\mathcal{F}}$ is not pseudoeffective, while

- if $K_{\mathcal{F}}$ is pseudoeffective with Zariski decomposition $K_{\mathcal{F}}=P+N$, then $\nu(\mathcal{F})=0$ if $P$ is numerically zero, $\nu(\mathcal{F})=1$ if $P \neq 0$ but $P^{2}=0$, and $\nu(\mathcal{F})=2$ if $P^{2}>0$.

The classification of foliations with negative numerical Kodaira dimension stated in the next result is due to Miyaoka.

Theorem 2.6. Let $\mathcal{F}$ be a foliation on a projective surface $X$. If $K_{\mathcal{F}}$ is not pseudoeffective, then $\mathcal{F}$ is birationally equivalent to a $\mathbb{P}^{1}$-bundle over a curve.

\subsection{Relatively minimal models}

Definition 2.7. Let $\mathcal{F}$ be a foliation with canonical singularities on a smooth projective surface $X$. An irreducible curve $C \subset X$ is called $\mathcal{F}$-exceptional if $K_{X} \cdot C=-1$ (that is, $C \simeq \mathbb{P}^{1}$ and $\left.C^{2}=-1\right)$ and the contraction of $C$ gives rise to a foliation with canonical singularities.

DeFinition 2.8. Let $\mathcal{F}$ be a foliation with canonical singularities on a smooth projective surface $X$. A relatively minimal model for $\mathcal{F}$ is the datum of a foliation $\mathcal{G}$ with canonical singularities and without $\mathcal{G}$-exceptional curves on a smooth projective surface $Y$ which is birationally equivalent to $\mathcal{F}$. We say that $\mathcal{G}$ is a minimal model if for any birational map $\pi: Z \rightarrow Y$ and any foliation $\mathcal{H}$ on $Z$ with canonical singularities such that $\pi_{*} \mathcal{H}=\mathcal{G}$, the map $\pi$ is a birational morphism.

The definitions above and the next result are essentially due to Brunella [Bru99]. The only minor difference is that in the original definition of $\mathcal{F}$-exceptional curve, Brunella considered only reduced singularities instead of canonical singularities. Nonetheless, his proof also works in this slightly more general situation.

THEOREM 2.9. Let $\mathcal{F}$ be a foliation with at worst canonical singularities on a smooth surface $X$. There exists a birational morphism $\pi: X \rightarrow Y$ such that $\pi_{*} \mathcal{F}$ is a relatively minimal model for $\mathcal{F}$. Moreover, $\pi_{*} \mathcal{F}$ is a minimal model for $\mathcal{F}$ unless $\mathcal{F}$ is birationally equivalent to a rational fibration, a Riccati foliation, or Brunella's special foliation $\mathcal{H}$.

The reader will find the explicit construction of the foliation $\mathcal{H}$ from the theorem in [Bru99]. Remark 2.10. Theorem 2.9 highlights one of the differences between the birational classification of projective surfaces and that of foliations on surfaces: while surfaces of non-negative Kodaira dimension always have a unique minimal model, there are foliations of Kodaira dimension zero and one which do not have unique minimal models. 


\section{J. V. PEREIRA AND R. Svaldi}

\subsection{Zariski decomposition and nef models}

If $\mathcal{L}$ is a pseudoeffective line bundle on a smooth projective surface, then $\mathcal{L}$ is $\mathbb{Q}$-linearly equivalent to $P_{\mathcal{L}}+N_{\mathcal{L}}$, where $P_{\mathcal{L}}$ is a nef $\mathbb{Q}$-divisor and $N_{\mathcal{L}}$ is a contractible effective $\mathbb{Q}$-divisor satisfying $P_{\mathcal{L}} \cdot N_{\mathcal{L}}=0$. This is the so-called Zariski decomposition of $\mathcal{L}$. We will denote by $\mathrm{i}(\mathcal{F})$ the index of $K_{\mathcal{F}}$, that is, the minimum of the set $\{n \in \mathbb{N} \mid n N$ has integral coefficients $\}$.

Theorem 2.11 ([Bru04, Chapter 8, Theorem 1]). Let $\mathcal{F}$ be a relatively minimal foliation on a smooth projective surface $X$. If $K_{\mathcal{F}}$ is pseudoeffective and $P+N$ is its Zariski decomposition, then the support of $N$ is a disjoint union of Hirzebruch-Jung strings.

A Hirzebruch-Jung string is a chain of smooth rational curves of self-intersection at most -2 . At one end of the chain, the handle of the Hirzebruch-Jung string, the foliation has only one singularity. Every other curve in the chain contains two singularities of the foliation. There is only one singularity of $\mathcal{F}$ on the Hirzebruch-Jung string which does not coincide with a singularity of its support. There exists a unique leaf of $\mathcal{F}$ not contained in the Hirzebruch-Jung string that passes through this singularity. Such a curve is called the tail of the Hirzebruch-Jung string.

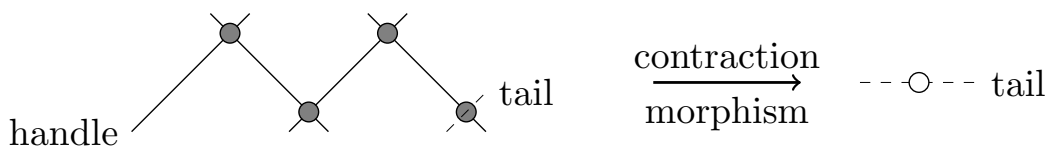

DeFinition 2.12. Let $\mathcal{F}$ be a relatively minimal foliation with pseudoeffective $K_{\mathcal{F}}$ on a smooth projective surface $X$. The order of a maximal Hirzebruch-Jung string contained in the support of $N$ is the determinant of the negative of the intersection matrix of its support.

The following proposition shows that the order and the index are closely related.

Proposition 2.13. We use the notation of Definition 2.12. The following assertions hold true:

(i) The order of a maximal Hirzebruch-Jung string $J$ contained in the support of $N$ coincides with the smallest $o \in \mathbb{N}$ such that the coefficients of $N$ corresponding to curves in $J$ belong to $(1 / o) \mathbb{N}$.

(ii) The contraction of a Hirzebruch-Jung string of order o is locally isomorphic to the quotient of a smooth foliation on $\left(\mathbb{C}^{2}, 0\right)$ by the cyclic group generated by an automorphism of the form $(x, y) \mapsto\left(\xi_{o} \cdot x, \xi_{o}^{a} \cdot y\right)$, where $\xi_{o}$ is a primitive root of unity of order $o$ and $a$ is a natural number relatively prime to $o$.

Proof. The statement is local, so we may very well assume that the support of $N$ is connected. Let us write $N=\sum_{i=1}^{k} a_{i} E_{i}$, where the $E_{i}$ are the irreducible components of $N$. We denote by $E_{1}$ the handle of the Hirzebruch-Jung string, while the other curves are numbered following the order in which they appear in the chain.

Let $A=\left(E_{i} \cdot E_{j}\right)_{i, j}$ be the intersection matrix of the Hirzebruch-Jung string, and let $o=$ $\operatorname{det}(-A)$ be the order of the Hirzebruch-Jung string. To determine the coefficients $a_{1}, \ldots, a_{k}$, we have to solve the linear system $(-A) \cdot\left(a_{1}, a_{2}, \ldots, a_{k}\right)^{\mathrm{T}}=(1,0, \ldots, 0)^{\mathrm{T}}$. Therefore, the coefficients $a_{i}$ certainly lie in $(1 / o) \mathbb{N}$. To see that $o$ is the minimal number with this property, it suffices to notice that $a_{k}=1 / o$; cf. [McQ08, proof of Proposition III.1.4]. This proves item (i). Item (ii) is [McQ08, Reinterpretation III.2.bis.3.a]

In the lemma below, we collect some properties of tails of Hirzebruch-Jung strings for later use. 


\section{EFFECTIVE ALGEBRAIC INTEGRATION IN BOUNDED GENUS}

LEMma 2.14. Let $\mathcal{F}$ be a relatively minimal foliation with pseudoeffective canonical bundle on a smooth projective surface $X$. Let $T$ be an irreducible invariant curve not contained in the support of $N$, and let $o_{1}, \ldots, o_{k}$ be the orders of Hirzebruch-Jung strings intersecting $T$. Then the following assertions hold true:

(i) The intersection of the positive part of the Zariski decomposition of $K_{\mathcal{F}}$ with $T$ is given by the formula

$$
P \cdot T=K_{\mathcal{F}} \cdot T-\sum_{i=1}^{k} \frac{1}{o_{i}} .
$$

(ii) If $\mathcal{F}$ admits a holomorphic first integral $f: U \rightarrow \mathbb{C}$ defined on an $\mathcal{F}$-invariant neighborhood of $T$ which vanishes along $T$, then the vanishing order along $T$ is a multiple of the least common multiple of $o_{1}, \ldots, o_{k}$.

Proof. Item (i) is [McQ08, Remark III.1.3.a]. To verify item (ii), let us work locally on a neighborhood $V$ of a Hirzebruch-Jung string intersecting $T$. Let $\pi: V \rightarrow W$ be the contraction of the Hirzebruch-Jung string we are considering and $o$ be its order. Perhaps after restricting $V$ to a smaller neighborhood, we can assume that $W$ is the quotient of a neighborhood $\tilde{V}$ of the origin in $\mathbb{C}^{2}$ by a cyclic group generated by $\varphi(x, y)=\left(\xi_{o} \cdot x, \xi_{o}^{a} \cdot y\right)$, according to Proposition 2.13. We can also assume that the pull-back $\mathcal{G}$ of $\pi_{*}\left(\mathcal{F}_{\mid V}\right)$ to $\tilde{V}$ is the foliation defined by the level sets of the coordinate function $y$. The pull-back of $\pi_{*}\left(f_{\mid V}\right)$ to $\tilde{V}$ is a holomorphic function $g$ constant along the leaves of $\mathcal{G}$. The $\varphi$-invariance of $g$ implies that $g(x, y)=h\left(y^{o}\right)$ for some one-variable holomorphic function $h$. Item (ii) follows.

DEFinition 2.15. Let $\mathcal{F}$ be a relatively minimal foliation on a smooth surface $X$ with pseudoeffective canonical divisor. The nef model of $\mathcal{F}$ is the foliation obtained by contracting the negative part of the Zariski decomposition of $K_{\mathcal{F}}$.

\subsection{Canonical models}

Definition 2.16. A foliation $\mathcal{F}$ on a normal projective surface $X$ is called a canonical model if $K_{\mathcal{F}}$ is nef and $K_{\mathcal{F}} \cdot C=0$ implies $C^{2} \geqslant 0$ for every irreducible curve $C \subset X$.

TheOREM 2.17 ([McQ08, Theorem 3.3.2]). Let $\mathcal{F}$ be relatively minimal foliation with $K_{\mathcal{F}}$ pseudoeffective on a smooth surface $X$. Then there exists a morphism $\pi: X \rightarrow Y$ from $X$ to a normal projective surface $Y$ such that $\mathcal{G}=\pi_{*} \mathcal{F}$ is a canonical model. The singular points of $Y$ and the corresponding exceptional fibers of $\pi$ are of one of the following forms:

(i) The singular point is a cyclic quotient singularity, and the exceptional divisor over it is a chain of rational curves of self-intersection at most -2 ,

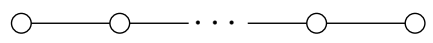

The foliation around the singular point is the quotient of a smooth foliation, or the quotient of a canonical foliation singularity on a (germ of a) smooth surface;

(ii) The singular point is a dihedral quotient singularity, and the exceptional divisor over it has the following dual graph:

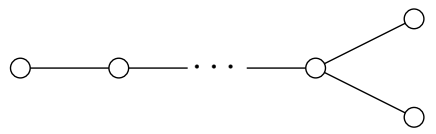

The foliation around the singularity is again the quotient of a smooth foliation or of a canonical singularity on a (germ of a) smooth surface. 


\section{J. V. Pereira And R. Svaldi}

(iii) The singular point is an elliptic Gorenstein singularity, and the exceptional divisor is a cycle of smooth rational curves each of self-intersection at most -2

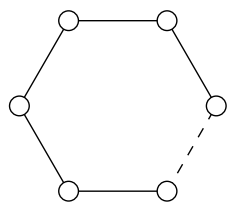

or a unique nodal rational curve of negative self-intersection. The foliation around the singular point is isomorphic to a cusp of a Hilbert modular foliation (cf. [McQ08, Theorem IV.2.2]). Moreover, the canonical bundle of the foliation on the canonical model is never $\mathbb{Q}$-Cartier.

When compared with the theory for projective surfaces, item (iii) of Theorem 2.17 is quite surprising. The fact that the canonical bundle is never $\mathbb{Q}$-Cartier is a clear obstruction to the base point freeness of $\left|K_{\mathcal{F}}^{\otimes n}\right|$ and to the finite generation of the canonical algebra of the foliation. It turns out that this is the only obstruction; cf. [McQ08, Corollary IV.2.3].

\subsection{Kodaira dimension zero}

TheOREm 2.18 ([McQ08, Fact IV.3.3]). Let $\mathcal{F}$ be a relatively minimal foliation on a smooth projective surface $X$ with $\nu(\mathcal{F})=0$. Let $\pi: X \rightarrow Z$ be the contraction of the negative part of $K_{\mathcal{F}}$; that is, $\pi_{*} \mathcal{F}$ is a nef model for $\mathcal{F}$. Then there exist a smooth projective surface $Y$ and a quasi-étale cyclic covering $p: Y \rightarrow Z$ of degree $\mathrm{i}(\mathcal{F})$ such that $p^{*} \pi_{*} \mathcal{F}$ is a foliation with trivial canonical bundle. In particular, $\operatorname{kod}(\mathcal{F})=0$.

The resulting surface $Y$ belongs to the following list:

(1) Product of a hyperbolic curve and an elliptic curve

(2) Abelian surface

(3) Projective bundle over an elliptic curve

(4) Rational surface

Consequently, the Kawamata log terminal (klt) surface $Z$ has Kodaira dimension 1 , 0 , or $-\infty$ according to whether $Y$ fits in case (1), (2), or (3)/(4). One can also determine the possibilities for the index of $\mathcal{F}$. This is done in [Per02]. There, it is shown that

$$
\mathrm{i}(\mathcal{F}) \in\{1,2,3,4,5,6,8,10,12\}
$$

when $\mathcal{F}$ has Kodaira dimension zero.

\subsection{Kodaira dimension one}

The classification of foliations of Kodaira dimension one is essentially due to Mendes; see [Men00, Theorem 3.3.1].

Theorem 2.19. Let $\mathcal{F}$ be a relatively minimal foliation on a smooth projective surface $X$. Assume that $\operatorname{kod}(\mathcal{F})=1$, and let $f: X \rightarrow C$ be the Iitaka fibration of $K_{\mathcal{F}}$. If $\mathcal{F}$ coincides with the foliation defined by $f$, then $f$ is a non-isotrivial elliptic fibration. Otherwise, $\mathcal{F}$ is completely transverse to a general fiber $F$ of $f$, and we have the following possibilities:

(i) The genus of $F$ is zero, and $\mathcal{F}$ is a Riccati foliation.

(ii) The genus of $F$ is one, and $\mathcal{F}$ is a turbulent foliation.

(iii) The genus of $F$ is at least two, and $\mathcal{F}$ is an isotrivial fibration of genus at least two. 


\section{EFFECTIVE ALGEBRAIC INTEGRATION IN BOUNDED GENUS}

\subsection{Non-abundant foliations}

The most striking difference between the birational classification of projective surfaces and the classification of rank one foliations in dimension two is the existence of foliations having canonical bundle with numerical dimension one and negative Kodaira dimension. This phenomenon is restricted to a rather special class of foliations, as pointed out by the next result.

THEOREM 2.20. Let $\mathcal{F}$ be a relatively minimal foliation on a smooth projective surface $X$. If the numerical dimension of $\mathcal{F}$ does not coincide with the Kodaira dimension of $\mathcal{F}$, then

(i) $\nu(\mathcal{F})=1$,

(ii) $\operatorname{kod}(\mathcal{F})=-\infty$,

(iii) $X$ is the minimal desingularization of the Baily-Borel compactification of an irreducible quotient of $\mathbb{H} \times \mathbb{H}$, and

(iv) $\mathcal{F}$ is induced by one of the two natural fibrations on $\mathbb{H} \times \mathbb{H}$.

Arguably, this result constitutes the hardest part of the classification of foliations. The known proofs of this result rely heavily on Brunella's plurisubharmonic variation of the Poincaré metric and were obtained by Brunella and McQuillan in a collaborative effort.

In Section 6, we will carry out a classification of foliations in terms of another birational invariant. This new classification relies heavily on the original classification of surface foliations according to their Kodaira dimension; nonetheless, it does not need the full power of it. In particular, all that we need to know about non-abundant foliations is contained in the following lemma.

Lemma 2.21. Let $\mathcal{F}$ be a relatively minimal foliation with $\nu(\mathcal{F})=1$ and $\operatorname{kod}(\mathcal{F})=-\infty$. Then $h^{1}\left(X, \mathcal{O}_{X}\right)=0$ and $P \cdot N_{\mathcal{F}}^{*}=P \cdot K_{X}>0$, where $P$ is the positive part of the Zariski decomposition of $K_{\mathcal{F}}$.

Proof. If $h^{1}\left(X, \mathcal{O}_{X}\right)=h^{0}\left(X, \Omega_{X}^{1}\right) \neq 0$, then the restriction of a holomorphic 1-form to the leaves of $\mathcal{F}$ either vanishes identically or gives rise to a non-zero section of $K_{\mathcal{F}}$. Thus if $\operatorname{kod}(\mathcal{F})=-\infty$, we obtain that $\mathcal{F}$ factors through the Albanese map of $X$ and is a fibration. Hence $\operatorname{kod}(\mathcal{F}) \geqslant 0$, contrary to our assumptions. Thus $h^{1}\left(X, \mathcal{O}_{X}\right)=0$.

Since $h^{1}\left(X, \mathcal{O}_{X}\right)=0$, we obtain that $\chi\left(\mathcal{O}_{X}\right) \geqslant 1$. Let $\mathcal{L}=\mathcal{O}_{X}(m P)$, where $m$ is a sufficiently divisible positive integer. By the Riemann-Roch theorem,

$$
\chi(\mathcal{L})=\chi\left(\mathcal{O}_{X}\right)+\frac{1}{2}\left(m^{2} P^{2}-m P \cdot K_{X}\right) .
$$

If $P \cdot K_{X}<0$, then $\chi(\mathcal{L})>0$. Thus $h^{0}(X, \mathcal{L})+h^{2}(X, \mathcal{L})>0$. But if $m$ is sufficiently large, then $K_{X} \otimes \mathcal{L}^{*}$ is not pseudoeffective, and consequently $h^{2}(X, \mathcal{L})=h^{0}\left(X, K_{X} \otimes \mathcal{L}^{*}\right)=0$. It follows that $h^{0}\left(X, K_{\mathcal{F}}^{\otimes m}\right)=h^{0}(X, \mathcal{L})>0$, contradicting $\operatorname{kod}(\mathcal{F})=-\infty$.

\section{Effective threshold and adjoint dimension}

In this section, we define the effective threshold and the adjoint dimension of a foliation on a smooth projective surface and prove their birational invariance.

\subsection{Effective threshold}

Definition 3.1. Let $\mathcal{F}$ be a foliation with canonical singularities on a smooth projective surface $X$. If the canonical bundle of $\mathcal{F}$ is pseudoeffective, then we define the effective threshold 


\section{J. V. Pereira and R. Svaldi}

$\operatorname{eff}(\mathcal{F})$ of $\mathcal{F}$ as the largest $\varepsilon \in \mathbb{R}_{\geqslant 0} \cup\{\infty\}$ such that $K_{\mathcal{F}}+\varepsilon N_{\mathcal{F}}^{*}$ is pseudoeffective. If $K_{\mathcal{F}}$ is not pseudoeffective, then we set $\operatorname{eff}(\mathcal{F})=-\infty$.

Example 3.2. Let $\mathcal{F}$ be a very general foliation on $\mathbb{P}^{2}$ of degree $d$. It is well known that $\mathcal{F}$ has reduced, and in particular canonical, singularities. Recall that the degree of $\mathcal{F}$ is defined as the number of tangencies between $\mathcal{F}$ and a general line. In this case, $K_{\mathcal{F}}=\mathcal{O}_{\mathbb{P}^{2}}(d-1)$ and $N_{\mathcal{F}}^{*}=\mathcal{O}_{\mathbb{P}^{2}}(-d-2)$. If $d=0$, then $K_{\mathcal{F}}$ is not pseudoeffective. If instead $d \geqslant 1$, then $K_{\mathcal{F}}$ is pseudoeffective and

$$
\operatorname{eff}(\mathcal{F})=\frac{d-1}{d+2}
$$

The reader should notice that $\operatorname{eff}(\mathcal{F})<1$ for every foliation on $\mathbb{P}^{2}$.

This is by no means a coincidence since $K_{X}=K_{\mathcal{F}}+N_{\mathcal{F}}^{*}$ and foliations on a surface $X$ of negative Kodaira dimension will always have $\operatorname{eff}(\mathcal{F})<1$ as $K_{X}$ is not pseudoeffective. If instead $X$ has non-negative Kodaira dimension, then $K_{X}$ is pseudoeffective, and consequently $\operatorname{eff}(\mathcal{F}) \geqslant 1$ for every foliation on $X$.

Similarly, one sees that $\operatorname{eff}(\mathcal{F})=\infty$ if and only if both $K_{\mathcal{F}}$ and $N_{\mathcal{F}}^{*}$ are pseudoeffective. Foliations with pseudoeffective conormal bundle have recently been classified by Touzet [Tou16]. They fit into one of the following descriptions:

(1) After a finite étale cover, $\mathcal{F}$ is defined by a closed holomorphic 1-form.

(2) There exists a morphism from $X$ to a quotient of a polydisc $\mathbb{D}^{m}$ by an irreducible lattice, and $\mathcal{F}$ is the pull-back of one of the $m$ tautological foliations on the polydisc. In particular, $\mathcal{F}$ is transversely hyperbolic.

Notice that the dimension of the ambient manifold is not necessarily equal to the dimension of the polydisc.

Remark 3.3. Using the identity $K_{X}=K_{\mathcal{F}}+N_{\mathcal{F}}^{*}$, we can write

$$
K_{\mathcal{F}}+\varepsilon N_{\mathcal{F}}^{*}=(1-\varepsilon)\left(K_{\mathcal{F}}+\frac{\varepsilon}{1-\varepsilon} K_{X}\right)
$$

when $\varepsilon \neq 1$.

When $\operatorname{eff}(\mathcal{F})$ is small, we will often work with $K_{\mathcal{F}}+\varepsilon K_{X}$ as that is more convenient.

\subsection{Adjoint dimension}

Definition 3.4. Let $\mathcal{F}$ be a foliation with canonical singularities on a projective surface $X$. Consider the pluricanonical maps

$$
\varphi_{m, n}: X \rightarrow \mathbb{P} H^{0}\left(X, K_{\mathcal{F}}^{\otimes m} \otimes N_{\mathcal{F}}^{* \otimes n}\right)^{*}
$$

for $m \geqslant 1$ and $n \geqslant 1$. The adjoint dimension of $\mathcal{F}$, denoted by $\operatorname{adj}(\mathcal{F})$, is the maximal dimension of the image of these maps. If $h^{0}\left(X, K_{\mathcal{F}}{ }^{\otimes m} \otimes N_{\mathcal{F}}^{*}{ }^{\otimes n}\right)=0$ for every $m, n \geqslant 1$, then we set $\operatorname{adj}(\mathcal{F})=-\infty$.

Definition 3.5. Let $\mathcal{F}$ be a foliation with canonical singularities on a projective surface $X$. The numerical adjoint dimension $\operatorname{adj}_{\text {num }}(\mathcal{F})$ of $\mathcal{F}$ is equal to $-\infty$ if $\operatorname{eff}(\mathcal{F}) \leqslant 0$ and equal to the maximal numerical dimension of $K_{\mathcal{F}}+\varepsilon N_{\mathcal{F}}^{*}$ for $\varepsilon \in(0, \operatorname{eff}(\mathcal{F}))$ otherwise.

Of course, $\operatorname{adj}(\mathcal{F}) \leqslant \operatorname{adjnum}(\mathcal{F})$. 


\section{EFFECTIVE ALGEBRAIC INTEGRATION IN BOUNDED GENUS}

\subsection{Birational invariance}

The significance of the concepts of effective threshold and of (numerical) adjoint dimension for the purpose of the birational classification of foliations on surfaces is assured by the next proposition.

Proposition 3.6. Let $(X, \mathcal{F})$ and $(Y, \mathcal{G})$ be two birationally equivalent foliations. If $\mathcal{F}$ and $\mathcal{G}$ have at worst canonical singularities, then $\operatorname{eff}(\mathcal{F})=\operatorname{eff}(\mathcal{G}), \operatorname{adj}(\mathcal{F})=\operatorname{adj}(\mathcal{G})$, and $\operatorname{adj}$ num $(\mathcal{F})=$

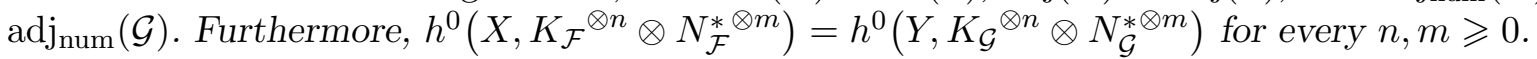

Proof. Since we can choose a foliation $(Z, \mathcal{H})$ on a smooth projective surface $Z$ dominating both $(X, \mathcal{F})$ and $(Y, \mathcal{G})$, there is no loss of generality in assuming the existence of a birational morphism $\pi:(X, \mathcal{F}) \rightarrow(Y, \mathcal{G})$. In fact, we can (and will) even assume that $\pi$ is the blow-up of a point $p \in Y$. Let $E$ be the exceptional divisor.

We will first prove that $\operatorname{eff}(\mathcal{F})=\operatorname{eff}(\mathcal{G})$. First, notice that $K_{\mathcal{G}}+\varepsilon N_{\mathcal{G}}^{*}=\pi_{*}\left(K_{\mathcal{F}}+\varepsilon N_{\mathcal{F}}^{*}\right)$. Therefore, if $K_{\mathcal{F}}+\varepsilon N_{\mathcal{F}}^{*}$ is pseudoeffective, then the same holds true for $K_{\mathcal{G}}+\varepsilon N_{\mathcal{G}}^{*}$. This shows that $\operatorname{eff}(\mathcal{G}) \geqslant \operatorname{eff}(\mathcal{F})$. To prove the converse inequality, we will need to use that $\mathcal{G}$ has canonical singularities. Since $\pi$ is the blow-up of a point by assumption, we have that $K_{\mathcal{F}}-\pi^{*} K_{\mathcal{G}}=a E$ for some $a \in\{0,1\}$. Since $K_{X}-\pi^{*} K_{Y}=E$, we also have that $N_{\mathcal{F}}^{*}-\pi^{*} N_{\mathcal{G}}^{*}=(1-a) E$, and consequently

$$
K_{\mathcal{F}}+\varepsilon N_{\mathcal{F}}^{*}=\pi^{*}\left(K_{\mathcal{G}}+\varepsilon N_{\mathcal{G}}^{*}\right)+(a+\varepsilon(1-a)) E .
$$

Therefore, if $K_{\mathcal{G}}+\varepsilon N_{\mathcal{G}}^{*}$ is pseudoeffective, then the same holds true for $K_{\mathcal{F}}+\varepsilon N_{\mathcal{F}}^{*}$. We conclude that $\operatorname{eff}(\mathcal{G}) \leqslant \operatorname{eff}(\mathcal{F})$, and the equality between the effective thresholds follow.

Let us now prove that $\operatorname{adj}_{\text {num }}(\mathcal{F})=\operatorname{adj}_{\text {num }}(\mathcal{G})$. We have just seen that $\operatorname{eff}(\mathcal{F})=\operatorname{eff}(\mathcal{G})$. Then, $\operatorname{adj}_{\text {num }}(\mathcal{F})=-\infty$ if and only if $\operatorname{adj}_{\text {num }}(\mathcal{G})=-\infty$, by definition. Let us consider $\varepsilon \in(0, \operatorname{eff}(\mathcal{F}))$, and let

$$
\begin{gathered}
K_{\mathcal{F}}+\varepsilon N_{\mathcal{F}}^{*}=P_{\mathcal{F}, \varepsilon}+N_{\mathcal{F}, \varepsilon}, \\
K_{\mathcal{G}}+\varepsilon N_{\mathcal{G}}^{*}=P_{\mathcal{G}, \varepsilon}+N_{\mathcal{G}, \varepsilon}
\end{gathered}
$$

be the Zariski decompositions of the divisors under scrutiny. By [Pro03, Theorem 2.2] and (3.1), it follows that for any $\varepsilon \in(0$, eff $(\mathcal{F}))$, we have that $P_{\mathcal{F}, \varepsilon}=\pi^{*} P_{\mathcal{G}, \varepsilon}$ and $N_{\mathcal{F}, \varepsilon}=\pi^{*} N_{\mathcal{G}, \varepsilon}+a+\varepsilon(1-a)$. Since by definition $\operatorname{adj}_{\text {num }}(\mathcal{F})=\max \left\{\nu\left(P_{\mathcal{F}, \varepsilon}\right) \mid \varepsilon \in(0, \operatorname{eff}(\mathcal{F}))\right\}$ (and analogously for adj $\operatorname{lnm}_{\text {num }}(\mathcal{G})$ ), we have verified the desired equality.

To conclude the proof of the proposition, it suffices to verify that $h^{0}\left(X, K_{\mathcal{F}}{ }^{\otimes n} \otimes N_{\mathcal{F}}^{*}{ }^{\otimes m}\right)=$ $h^{0}\left(Y, K_{\mathcal{G}}{ }^{\otimes n} \otimes N_{\mathcal{G}}^{*}{ }^{\otimes m}\right)$ for every $n, m \geqslant 0$. Once these equalities are proved, the equality adj $(\mathcal{F})=$ $\operatorname{adj}(\mathcal{G})$ follows. Let us fix $n, m \geqslant 0$. From the isomorphism $K_{\mathcal{F}}{ }^{\otimes n} \otimes N_{\mathcal{F}}^{*} \otimes m=\pi^{*}\left(K_{\mathcal{G}}{ }^{\otimes n} \otimes N_{\mathcal{G}}^{* \otimes m}\right) \otimes$ $\mathcal{O}_{X}((n a+m(1-a)) E)$, we deduce the short exact sequence

$$
0 \rightarrow \pi^{*}\left(K_{\mathcal{G}}^{\otimes n} \otimes N_{\mathcal{G}}^{* \otimes m}\right) \rightarrow K_{\mathcal{F}}^{\otimes n} \otimes N_{\mathcal{F}}^{*}{ }^{\otimes m} \rightarrow \mathcal{O}_{E}((n a+m(1-a)) E) \rightarrow 0 .
$$

Since $h^{0}\left(E, \mathcal{O}_{E}((n a+(1-a)) E)=0\right.$, we obtain the sought identity.

\subsection{Convention}

For an arbitrary foliation $\mathcal{F}$ on a smooth projective surface $X$, we define the adjoint dimension, the numerical adjoint dimension, and the effective threshold as the corresponding quantity for any foliation $\mathcal{G}$ with canonical singularities that is birationally equivalent to $\mathcal{F}$. 


\section{J. V. PeReira AND R. Svaldi}

\section{Singularities}

\subsection{Adjoint discrepancy and $\varepsilon$-canonical singularities}

Definition 4.1. Let $\mathcal{F}$ be a foliation on $X$, and let $\pi: Y \rightarrow X$ be a birational morphism. Denote by $\mathcal{G}$ the pull-back of $\mathcal{F}$ under $\pi$. If $E$ is an exceptional divisor of $\pi$, then the adjoint discrepancy of $\mathcal{F}$ along $E$ is the function

$$
\begin{aligned}
a(\mathcal{F}, E):[0, \infty) & \longrightarrow \mathbb{R}, \\
t & \longrightarrow \operatorname{ord}_{E}\left(K_{\mathcal{G}}+t N_{\mathcal{G}}^{*}-\left(\pi^{*} K_{\mathcal{F}}+t \pi^{*} N_{\mathcal{F}}^{*}\right)\right) .
\end{aligned}
$$

Definition 4.2. Let $\mathcal{F}$ be a foliation on $X$ and $\varepsilon \geqslant 0$ a real number. A point $x \in X$ is $\varepsilon$-canonical if and only if the adjoint discrepancy of $\mathcal{F}$ along any divisor $E$ over $x$ satisfies $a(\mathcal{F}, E)(t) \geqslant 0$ for every $t \geqslant \varepsilon$. The foliation $\mathcal{F}$ is said to have $\varepsilon$-canonical singularities if every point $x \in X$ is $\varepsilon$-canonical. The smallest $\varepsilon$ for which $x \in X$ is $\varepsilon$-canonical will be called the canonical threshold of $\mathcal{F}$ at $x$.

Proposition 4.3. Let $(X, \mathcal{F})$ and $(Y, \mathcal{G})$ be two foliations on smooth projective surfaces. Assume that $\mathcal{F}$ and $\mathcal{G}$ are birationally equivalent. If both $\mathcal{F}$ and $\mathcal{G}$ have $\varepsilon$-canonical singularities, then for any pair of integers $n, m$ satisfying $m / n \geqslant \varepsilon$, we have that

$$
h^{0}\left(X, K_{\mathcal{F}}^{\otimes n} \otimes N_{\mathcal{F}}^{* \otimes m}\right)=h^{0}\left(Y, K_{\mathcal{G}}^{\otimes n} \otimes N_{\mathcal{G}}^{* \otimes m}\right) .
$$

In particular, if $\operatorname{eff}(\mathcal{F}) \geqslant \varepsilon$, then $\operatorname{eff}(\mathcal{F})=\operatorname{eff}(\mathcal{G})$.

Proof. The proof is completely analogous to the proof of Proposition 3.6.

Remark 4.4. We point out that $\varepsilon^{\prime}$-canonical singularities are $\varepsilon$-canonical for every $\varepsilon \geqslant \varepsilon^{\prime}$. In particular, canonical singularities are $\varepsilon$-canonical singularities for every $\varepsilon \geqslant 0$. Also note that the canonical threshold of a log canonical singularity is at most $\frac{1}{2}$; that is, log canonical singularities are $\varepsilon$-canonical for every $\varepsilon \geqslant \frac{1}{2}$. This is a straightforward consequence of the simple fact that for every divisor $E$ exceptional over $X$ extracted on a smooth birational surface $\pi: Y \rightarrow X$, we have that $\operatorname{ord}_{E}\left(K_{Y}-\pi^{*} K_{X}\right) \in \mathbb{Z}_{>0}$.

Notation 4.5 . If $p, q \geqslant 1$ are relatively prime integers, then we will write

$$
\frac{p}{q}=\left[u_{0}, u_{1}, \ldots, u_{n}\right]=u_{0}+\frac{1}{u_{1}+\frac{1}{\cdots+\frac{1}{u_{n}}}}
$$

for the continued fraction presentation of their quotient.

If $p, q \geqslant 1$ are relatively prime positive integers, then vector fields of the form $v=p x \partial / \partial x+$ $q y \partial / \partial y$ are tangent to the fibers of rational map $(x, y) \mapsto y^{p} / x^{q}$. As a simple computation shows, see for instance [MM80, Appendix 1, Theorem 3], the minimal reduction of singularities of $v$ coincides with the minimal resolution of indeterminacies of the rational map above by blowups on closed points. In particular, there exists a unique irreducible component of the exceptional divisor which is not invariant by the resulting foliation.

DeFinition 4.6. Let $p, q \geqslant 1$ be relatively prime positive integers, and consider the germ of foliation on $X=\left(\mathbb{C}^{2}, 0\right)$ defined by $v=p x \partial / \partial x+q y \partial / \partial y$. Let $\pi: Y \rightarrow X$ be the minimal reduction of singularities of $\mathcal{F}$, let $\mathcal{G}$ be the transformed foliation $\pi^{*} \mathcal{F}$, and let $E$ be the irreducible component of the exceptional divisor which is not $\mathcal{G}$ invariant. We will denote the order of $K_{Y}-\pi^{*} K_{X}$ along $E$ by $\varphi(p, q)$. 


\section{EFFECTIVE ALGEBRAIC INTEGRATION IN BOUNDED GENUS}

Lemma 4.7. We use the notation of Definition 4.6. If we write $p / q=\left[u_{0}, u_{1}, \ldots, u_{n}\right]$, then the following assertions hold true:

(i) The morphism $\pi$ is the composition of exactly $\sum_{i=0}^{n} u_{i}$ blow-ups.

(ii) The order of $K_{Y}-\pi^{*} K_{X}$ along $E$ satisfies $\varphi(p, q) \geqslant \sum_{i=0}^{n} u_{i}$.

Proof. The key observation is that the reduction of singularities of $v$ follows step-by-step Euclid's algorithm for the computation of $\operatorname{gcd}(p, q)$. Assume $p \geqslant q$, and write $p / q$ as a continued fraction $\left[u_{0}, u_{1}, \ldots, u_{n}\right]$. The proof will be by induction on the number $N=\sum_{i=1}^{n} u_{i}$.

If $p=q=1$, then clearly $N=1$, and the result is obvious in this case. Assume $p>q$, and consider the blow-up $s: Z \rightarrow X$ of the origin with exceptional divisor $E_{0}$. Over the exceptional divisor, we will find two singularities with eigenvalues $(p-q, q)$ and $(p, q-p)$. Since we are assuming $p>q$, the pair $(p, q-p)$ corresponds to a canonical singularity, while the pair $(p-q, q)$ corresponds to a non-canonical singularity. Observe that

$$
\frac{p-q}{q}=\left[u_{0}-1, u_{1}, \ldots, u_{n}\right] .
$$

Assuming that the result is true for $N-1$, the first part of the statement follows.

To verify item (ii), notice that $K_{Z}=s^{*} K_{X}+E_{0}$. If $r: Y \rightarrow Z$ is the minimal desingularization of $s^{*} \mathcal{F}$, then by induction hypothesis, $\operatorname{ord}_{E}\left(K_{Y}-r^{*} K_{Z}\right) \geqslant N-1$. Since $\pi=s \circ r$, we can write

$$
\begin{aligned}
\operatorname{ord}_{E}\left(K_{Y}-\pi^{*} K_{X}\right) & =\operatorname{ord}_{E}\left(K_{Y}-r^{*}\left(K_{Z}-E_{0}\right)\right) \\
& =\operatorname{ord}_{E}\left(K_{Y}-r^{*} K_{Z}\right)+\operatorname{ord}_{E}\left(r^{*} E_{0}\right) \geqslant N .
\end{aligned}
$$

The lemma then follows by induction.

Remark 4.8. The inequality in part (ii) of Lemma 4.7 becomes an equality only for singularities with eigenvalues of the form $(1, q)$. If $p$ and $q$ are both strictly greater than one, at some intermediate step, we will be forced to blow up at the intersection of two exceptional divisors and one will get a greater order at the end. For instance, if $p / q=\left[u_{0}, u_{1}\right]$, then the order of $K_{Y}-\pi^{*} K_{X}$ along the last exceptional divisor is $\varphi(p, q)=\left(u_{1}+1\right) u_{0}-1$.

As a consequence of the above description, we are able to characterize $\varepsilon$-canonical singularities for small values of $\varepsilon>0$.

Proposition 4.9. Let $\mathcal{F}$ be a germ of foliation on $\left(\mathbb{C}^{2}, 0\right)$. If the canonical threshold of $\mathcal{F}$ at 0 is strictly less than $1 / 4$, then 0 is a $\log$ canonical singularity.

Proof. Let $v$ be a generator of $T_{\mathcal{F}}$. First, assume that the linear part of $v$ is zero. If $\pi: Y \rightarrow\left(\mathbb{C}^{2}, 0\right)$ is the blow-up of the origin, $\mathcal{G}=\pi^{*} \mathcal{F}$, and $E$ is the exceptional divisor, then $K_{\mathcal{G}}=\pi^{*} K_{\mathcal{F}}-a E$, where $a \geqslant 1$. On the other hand, $N_{\mathcal{G}}^{*}=\pi^{*} N_{\mathcal{F}}^{*}+(a+1) E$. Therefore, if $\varepsilon<\frac{1}{2}$, then the origin is not $\varepsilon$-canonical.

Now, assume that the linear part of $v$ is non-zero but nilpotent. We will use the description of the resolution process of this kind of singularities presented in [Bru04, Chapter 1, proof of Theorem 1]. If we blow up the origin, then we obtain only one singularity over the exceptional divisor, which is invariant by the transformed foliation. This new singularity can have zero linear part or non-zero but nilpotent linear part. Let us analyze the two possibilities. Start with the case where the linear part is zero, and let $\pi: Y \rightarrow\left(\mathbb{C}^{2}, 0\right)$ be the composition of the two obvious blow-ups. As before, we will set $\mathcal{G}=\pi^{*} \mathcal{F}$ and will let $E_{1}$ and $E_{2}$ be the two irreducible components of the exceptional divisor of $\pi$, with $E_{2}$ corresponding to the last blow-up. Notice 


\section{J. V. Pereira and R. Svaldi}

that $K_{\mathcal{G}}=\pi^{*} K_{\mathcal{F}}-a E_{2}$ for some $a \geqslant 1$ and $N_{\mathcal{G}}^{*}=\pi^{*} N_{\mathcal{F}}^{*}+E_{1}+(a+2) E_{2}$. Hence if $\varepsilon<1 / 3$, then 0 is not an $\varepsilon$-canonical singularity. Let us now deal with the second possibility. If the blow-up of a nilpotent singularity with non-zero linear part leads to another nilpotent singularity with nonzero linear part, then one further blow-up gives rise to a singularity with trivial linear part. Now, let $\pi: Y \rightarrow\left(\mathbb{C}^{2}, 0\right)$ be the composition of the three obvious blow-ups, and let $E_{1}, E_{2}, E_{3}$ be the irreducible components of the exceptional divisor numbered according to the order of appearance. If we set $\mathcal{G}=\pi^{*} \mathcal{F}$, then $K_{\mathcal{G}}=\pi^{*} K_{\mathcal{F}}-a E_{3}$ for some $a \geqslant 1$ and $N_{\mathcal{G}}^{*}=\pi^{*} N_{\mathcal{F}}^{*}+E_{1}+2 E_{2}+(a+3) E_{3}$. Thus if $\varepsilon<1 / 4$, then 0 is not an $\varepsilon$-canonical singularity.

Therefore, if $\varepsilon<1 / 4$, then the linear part of $v$ is non-nilpotent, and we can apply [McQ08, Fact I.1.8] to conclude that 0 is a $\log$ canonical singularity of $\mathcal{F}$.

Corollary 4.10. Let $\mathcal{F}$ be a germ of foliation on $\left(\mathbb{C}^{2}, 0\right)$ defined by a germ of vector field $v$. If $0<\varepsilon<1 / 4$, then 0 is an $\varepsilon$-canonical singularity of $\mathcal{F}$ if and only if the linear part of $v$ is non-nilpotent and one of the following holds:

(i) The singularity of $v$ is canonical.

(ii) The singularity of $v$ is not canonical, $v$ is analytically conjugated to $p x \partial / \partial x+q y \partial / \partial y$ with $p$ and $q$ relatively prime positive integers, and $\varphi(p, q) \geqslant(1-\varepsilon) / \varepsilon$.

Proof. Proposition 4.9 implies that the linear part of $v$ is non-nilpotent. If 0 is not a canonical singularity, then by [McQ08, Fact I.1.9], we know that $v$ is analytically conjugated to $p x \partial / \partial x+$ $q y \partial / \partial y$ for suitable relatively prime positive integers $p$ and $q$. If $\pi: Y \rightarrow X=\left(\mathbb{C}^{2}, 0\right)$ is the minimal reduction of singularities of $\mathcal{F}$ and $E$ denotes the last exceptional divisor, then for $\mathcal{G}=\pi^{*} \mathcal{F}$, we have that $K_{\mathcal{G}}=\pi^{*} K_{\mathcal{F}}-E$. Therefore, the adjoint discrepancy of $\mathcal{F}$ along $E$ is (cf. Remark 3.3)

$$
\begin{aligned}
a(\mathcal{F}, E)(t) & =(1-t) \operatorname{ord}_{E}\left(K_{\mathcal{G}}+\frac{t}{1-t} K_{Y}-\pi^{*}\left(K_{\mathcal{F}}+\frac{t}{1-t} K_{X}\right)\right) \\
& =(1-t)\left(-1+\frac{t}{1-t} \varphi(p, q)\right) .
\end{aligned}
$$

Since the adjoint discrepancy is clearly non-negative along all the other divisors in the minimal resolution, it follows that 0 is an $\varepsilon$-canonical singularity if and only if $\varphi(p, q) \geqslant(1-\varepsilon) / \varepsilon$.

\subsection{Example: Log canonical foliations on the projective plane}

For a foliation $\mathcal{F}$ on the projective plane with log canonical singularities, one can easily verify the following assertions:

(1) If $d=\operatorname{deg}(\mathcal{F}) \geqslant 4$, then $\operatorname{eff}(\mathcal{F})=(d-1) /(d+2)$.

(2) If $d=\operatorname{deg}(\mathcal{F})=3$, then $\operatorname{eff}(\mathcal{F})=2 / 5$ unless $\mathcal{F}$ has radial singularities.

(3) If $d=\operatorname{deg}(\mathcal{F})=2$, then $\operatorname{eff}(\mathcal{F})=1 / 4$ unless $\mathcal{F}$ has radial singularities or dicritical singularities of type $(1,2)$.

One could try to pursue a case-by-case analysis in order to provide an explicit lower bound for the positive effective thresholds of foliations of degree two and three with log canonical singularities. We will show later in Section 7 that the positive effective thresholds of foliations varying in an algebraic family do not accumulate at zero. Unfortunately, our proof is not effective, and, a priori, the bound might depend on the family. 


\section{EFFECTIVE ALGEBRAIC INTEGRATION IN BOUNDED GENUS}

\section{Non-isotrivial fibrations}

\subsection{Producing sections}

Our original motivation to introduce and study the adjoint dimension of foliations lies in our poor understanding of the linear systems $\left|K_{\mathcal{F}}{ }^{\otimes n}\right|$. When $\mathcal{F}$ is a foliation of general type, we are not aware of lower bounds on $n$ such that $\left|K_{\mathcal{F}}{ }^{\otimes n}\right|$ is not empty. For the linear systems $\left|K_{\mathcal{F}}{ }^{\otimes n} \otimes K_{X}{ }^{\otimes m}\right|$, the situation is considerably better. We can apply the current knowledge on adjoint linear systems to obtain effective bounds on $n$ and $m$ such that $\left|K_{\mathcal{F}}{ }^{\otimes m} \otimes K_{X}{ }^{\otimes n}\right|$ defines a rational map with two-dimensional image.

Proposition 5.1. Let $\mathcal{F}$ be a foliation with canonical singularities on a smooth projective surface. If $\operatorname{kod}(\mathcal{F})=2$, then the linear system $\left|K_{X}+4 \mathrm{i}(\mathcal{F}) K_{\mathcal{F}}\right|$ defines a rational map with two-dimensional image.

Proof. This is an immediate consequence of [Rei88, Corollary 2].

Proposition 5.1 is certainly not optimal. The real question underlying the whole issue here is whether or not one can provide universal bounds which do not depend on the index of the foliation. The reader will find a more precise formulation of this question in Problem 6.8.

\subsection{Bound for the index of hyperbolic fibrations}

In order to use the results above to provide explicit bounds for the degree of leaves of non-isotrivial hyperbolic fibrations, we need to obtain bounds for the index of the foliation.

Lemma 5.2. Let $\mathcal{F}$ be a relatively minimal foliation on a smooth projective surface $X$. Assume that $K_{\mathcal{F}}$ is defined by a fibration $f: X \rightarrow C$ and that the general fiber of $f$ has genus at least two. If $T$ is an irreducible curve invariant by $\mathcal{F}$ which intersects the support of the negative part of $K_{\mathcal{F}}$ and is not contained in it (that is, $T$ is a tail), then one of the following holds:

(i) We have that $P \cdot T=0$, and $T$ intersects exactly two connected components of the support of $N$, both of them of order 2 .

(ii) $P \cdot T \geqslant 1 / 42$.

Proof. It follows from Lemma 2.14 that

$$
P \cdot T=K_{\mathcal{F}} \cdot T-\sum_{i=1}^{k} \frac{1}{o_{i}}=-\chi(T)+s+k-\sum_{i=1}^{k} \frac{1}{o_{i}},
$$

where $s$ is the number of singularities of $\mathcal{F}$ on $T$ which are not contained in the support of $N$ [Bru04, Chapter 2, Proposition 3].

Assume that $P \cdot T=0$. If $s=0$, then we have the following possibilities for $k$ and $o=$ $\left(o_{1}, \ldots, o_{k}\right): k=3$ and $o=(2,4,4)$; or $k=3$ and $o=(3,3,3)$; or $k=3$ and $o=(2,3,6)$; or $k=4$ and $o=(2,2,2,2)$. In all cases, the whole fiber $F$ containing $T$ is the union of $k$ Hirzebruch-Jung strings joined by a single common tail $T$ and $\chi(F)=\chi_{\text {orb }}(\tilde{T})=0$. Since $\chi(F)<0$ by assumption, we get that $P \cdot T>0$, contradicting our assumption. The only remaining possibility is $s=1$, $k=2$, and $o=(2,2)$. Item (i) follows.

If $P \cdot T>0$, then it is an elementary and well-known fact that the lower bound for (5.1) is equal to $1 / 42$ and is attained by $s=0, k=3$, and $o=(2,3,7)$. 


\section{J. V. Pereira And R. Svaldi}

Proposition 5.3. Let $\mathcal{F}$ be a relatively minimal foliation on a smooth projective surface $X$. Assume that $\mathcal{F}$ is defined by a fibration $f: X \rightarrow C$ and that the general fiber of $f$ has genus $g \geqslant 2$. Then

$$
\mathrm{i}(\mathcal{F}) \leqslant(42(2 g-2)) ! .
$$

Proof. Let $F=\sum m_{i} C_{i}$ be a fiber of $f$, and let $K_{\mathcal{F}}=P+N$ be the Zariski decomposition of $K_{\mathcal{F}}$. If $C_{i}$ is a tail, then according to Lemma 5.2, either the Hirzebruch-Jung strings intersecting it have order two, or $P \cdot C_{i} \geqslant 1 / 42$. In the latter case, we get that $m_{i} \leqslant 42(2 g-2)$ since $P \cdot F=K_{\mathcal{F}} \cdot F=-\chi(F)=2 g-2$. Moreover, Lemma 2.14(ii) implies that the least common multiple of the orders of the Hirzebruch-Jung strings intersecting $C_{i}$ divides $m_{i} \leqslant 42(2 g-2)$. The proposition is then proved.

\subsection{Boundedness of fibers of non-isotrivial fibrations of a given genus}

Theorem A of the introduction will follow rather easily from the more general result below.

THEOREM 5.4. Let $\mathcal{F}$ be a foliation with canonical singularities on a smooth projective surface $X$. Suppose that $\mathcal{F}$ is a fibration with general fiber $F$ of genus $g$. If $\operatorname{kod}(\mathcal{F})=2$, then for every nef divisor $H$, we have that

$$
F \cdot H \leqslant M\left(K_{X}+4 \mathrm{i}(\mathcal{F}) K_{\mathcal{F}}\right) \cdot H
$$

where $M=M(g)$ satisfies the inequality

$$
M \leqslant 2(4 \mathrm{i}(\mathcal{F})+1)(2 g-2) \leqslant(4(42(2 g-2)) !+1)(4 g-4) .
$$

Proof. When $\mathcal{F}$ is induced by a fibration and $\operatorname{kod}(\mathcal{F})=2$, the fibration is non-isotrivial, and the genus of the general fiber is at least 2; see [Ser92, Theorem 2.1] and the discussion before that theorem.

For simplicity, we will write $L=K_{X}+(4 \mathrm{i}(\mathcal{F})) K_{\mathcal{F}}$. Let $F$ denote a general leaf of $\mathcal{F}$. If $m \geqslant 1$ is an integer, then $\left.m L\right|_{F}=m(4 \mathrm{i}(\mathcal{F})+1) K_{F}$. On the one hand, the Riemann-Roch theorem implies that

$$
h^{0}\left(F, \mathcal{O}_{F}\left(\left.m L\right|_{F}\right)\right)=m(4 \mathrm{i}(\mathcal{F})+1)(2 g-2)-g+1 .
$$

On the other hand, since according to Proposition 5.1, the linear system $|L|$ defines a rational map with two-dimensional image, $h^{0}\left(X, \mathcal{O}_{X}(m L)\right) \geqslant\left(\begin{array}{c}m+2 \\ 2\end{array}\right)$.

If we take $m=2(4 \mathrm{i}(\mathcal{F})+1)(2 g(F)-2)$, then

$$
\begin{aligned}
h^{0}\left(X, \mathcal{O}_{X}(m L-F)\right) & \geqslant h^{0}\left(X, \mathcal{O}_{X}(m L)\right)-h^{0}\left(F, \mathcal{O}_{F}\left(m(4 \mathrm{i}(\mathcal{F})+1) K_{F}\right)\right. \\
& \geqslant\left(\begin{array}{c}
m+2 \\
2
\end{array}\right)-m(4 \mathrm{i}(\mathcal{F})+1)(2 g-2)+g-1=g .
\end{aligned}
$$

In particular, $|m L-F|$ is non-empty. As $H$ is an arbitrary nef divisor on $X$, we have that $(m L-F) \cdot H \geqslant 0$, which concludes the first part of the proof. The inequality in the last part of the statement is then just a consequence of Proposition 5.3.

\subsection{Proof of Theorem A}

Let $\mathcal{F}$ be a foliation on $\mathbb{P}^{2}$ birationally equivalent to a non-isotrivial fibration of genus $g \geqslant 2$. Notice that its canonical bundle is isomorphic to $\mathcal{O}_{\mathbb{P}^{2}}(\operatorname{deg}(\mathcal{F})-1)$. Let $\pi: X \rightarrow \mathbb{P}^{2}$ be a birational morphism such that all the singularities of $\mathcal{G}=\pi^{*} \mathcal{F}$ are canonical and also such that on this model, the foliation is induced by an actual fibration; that is, there exists a morphism $g: X \rightarrow \mathbb{P}^{1}$ with $\mathcal{G}=\operatorname{ker} d g$. 


\section{EFFECTIVE ALGEBRAIC INTEGRATION IN BOUNDED GENUS}

If we take $H=\pi^{*} \mathcal{O}_{\mathbb{P}^{2}}(1)$, then the degree of an algebraic leaf $L$ of $\mathcal{F}$ is given by

$$
\operatorname{deg}(L)=H \cdot \pi^{*} L=H \cdot \hat{L},
$$

where $\hat{L}$ is the strict transform of $L$. We can thus apply Theorem 5.4 and Proposition 5.3 to deduce that

$$
\begin{aligned}
\operatorname{deg}(L) & \leqslant(4(42(2 g-2)) !+1)(4 g-4)\left(K_{X}+4 \mathrm{i}(\mathcal{G}) K_{\mathcal{G}}\right) \cdot H \\
& =(4(42(2 g-2)) !+1)(4 g-4)\left(K_{\mathbb{P}^{2}}+4 \mathrm{i}(\mathcal{G}) K_{\mathcal{F}}\right) \cdot \pi_{*} H \\
& =(4(42(2 g-2)) !+1)(4 g-4)(-3+4(42(2 g-2)) !(\operatorname{deg}(\mathcal{F})-1)) \\
& \leqslant(4(42(2 g-2)) !)^{2}(4 g-4) \operatorname{deg}(\mathcal{F}) .
\end{aligned}
$$

This concludes the proof of Theorem A.

\subsection{Log canonical foliations on $\mathbb{P}^{2}$ of high degree}

The bounds appearing in Theorem 5.4 are ridiculously large and far from optimal. Proposition 5.5 below combined with the results presented in Section 7 (notably Theorem 7.6) indicates that the dependence of $M$ on $g$ in Theorem 5.4 should be at worst linear on $g$. The results of [McQ17] also indicate the existence of such linear bounds which are not universal but depend on the family of foliations in question.

Proposition 5.5. Let $\mathcal{F}$ be a foliation with canonical singularities on a projective surface $X$. Assume that $\mathcal{F}$ is a fibration with general fiber $F$ of geometric genus $g \geqslant 2$ and that $H^{0}\left(X, K_{\mathcal{F}}^{\otimes a} \otimes\right.$ $N_{\mathcal{F}}^{* \otimes b}$ ) admits three algebraically independent sections for some $a>0$ and $b \geqslant 0$. Then for every nef divisor $H$, we have that

$$
F \cdot H \leqslant 2 a(2 g-2)\left(a K_{\mathcal{F}}+b N_{\mathcal{F}}^{*}\right) \cdot H .
$$

Proof. For simplicity, we will write $\mathcal{L}=a K_{\mathcal{F}}+b N_{\mathcal{F}}^{*}$. Let $F$ denote a general leaf of $\mathcal{F}$. If $m \geqslant 1$ is an integer, then $m L_{\mid F}=a m K_{F}$. On the one hand, by the Riemann-Roch theorem,

$$
h^{0}\left(F, \mathcal{O}_{F}(m L)_{\mid F}\right)=m a(2 g-2)-g+1 .
$$

On the other hand, our assumption on $H^{0}\left(X, \mathcal{O}_{X}(L)\right)$ implies that $h^{0}\left(X, \mathcal{O}_{X}(m L)\right) \geqslant\left(\begin{array}{c}m+2 \\ 2\end{array}\right)$. If we take $m=2 a(2 g-2)$, then

$$
\begin{aligned}
h^{0}\left(X, \mathcal{O}_{X}(m L)\right)-h^{0}\left(F, \mathcal{O}_{F}\left(m L_{\mid F}\right)\right) & \geqslant\left(\begin{array}{c}
2 a(2 g-2)+2 \\
2
\end{array}\right)-2 a^{2}(2 g-2)^{2}+(g-1) \\
& =6 a(g-1)+g>0 .
\end{aligned}
$$

In particular, $|2 a(2 g-2) L-F|$ is non-empty.

If $H$ is an arbitrary nef divisor on $X$, then $(2 a(2 g-2) L-F) \cdot H \geqslant 0$, which concludes the proof.

In the case of foliations of the projective plane with log canonical singularities and of degree greater than or equal to five, we can actually obtain bounds that are better than linear using a simple variation of the argument used to prove Proposition 5.5.

Theorem 5.6. Let $\mathcal{F}$ be a foliation on $\mathbb{P}^{2}$ of degree $d \geqslant 5$. Assume that $\mathcal{F}$ has $\log$ canonical singularities and admits a rational first integral with general fiber of geometric genus $g \geqslant 2$. If $F$ is a general leaf of $\mathcal{F}$, then

$$
\operatorname{deg}(F) \leqslant\left\lceil\frac{4(2 g-2)}{(d-4)^{2}}\right\rceil(d-4)
$$




\section{J. V. Pereira And R. Svaldi}

Proof. Since the singularities of $\mathcal{F}$ are $\varepsilon$-canonical for $\varepsilon=\frac{1}{2}$ (see Remark 4.4), the dimension of the vector spaces $H^{0}\left(\mathbb{P}^{2}, K_{\mathcal{F}}^{\otimes 2 m} \otimes N_{\mathcal{F}}^{*} \otimes m\right)$ for $m>0$ is unaltered after we replace $\mathcal{F}$ with a model with at worst canonical singularities.

Let $F$ be a general fiber of the rational first integral of $\mathcal{F}$, and consider the real-valued function

$$
f(m)=\left(\begin{array}{c}
m(d-4)+2 \\
2
\end{array}\right)-2 m(2 g-2)+g-1 .
$$

Its values on positive integers correspond to the difference $h^{0}\left(\mathbb{P}^{2}, K_{\mathcal{F}}^{\otimes 2 m} \otimes N_{\mathcal{F}}^{*} \otimes m\right)-h^{0}\left(\tilde{F}, K_{\tilde{F}}^{\otimes 2 m}\right)$, where $\tilde{F}$ is the normalization of $F$. Since $f\left(4(2 g-2) /(d-4)^{2}\right)=(d g+8 g-12) /(d-4)$, which is clearly positive, and moreover the derivative of $f$ satisfies $f^{\prime}\left(4(2 g-2) /(d-4)^{2}\right)=$ $(3 / 2) d+4 g-10>0$, it follows that if $m$ is the smallest integer greater than $4(2 g-2) /(d-4)^{2}$, then there exists a section of $K_{\mathcal{F}}^{\otimes 2 m} \otimes N_{\mathcal{F}}^{*} \otimes m \simeq \mathcal{O}_{\mathbb{P}^{2}}(m(d-4))$ vanishing identically on $F$. The theorem follows.

As already mentioned in the introduction, Theorem 5.6 refines a classical result of Poincaré; see [Poi91, Des noeuds dicritiques, p. 169, and Des noeuds monocritiques, p. 176] and [Per03, Chapter 7, Corollary 14].

\section{Classification via the adjoint dimension}

In this section, we apply the results recalled in Section 2 to obtain a classification of foliations on surfaces according to their adjoint dimension.

\section{1 $K_{X}$-negative extremal rays}

Recall that for a smooth projective surface $X$, the $K_{X}$-negative extremal rays are spanned by numerical classes of rational curves of self-intersection $-1,0$, or 1 . The first case corresponds to the exceptional divisor of the blow-up of a smooth point, the second to a smooth fiber of a $\mathbb{P}^{1}$-bundle, while the last one is just the class of a line in $\mathbb{P}^{2}$.

LEMma 6.1. Let $\mathcal{F}$ be a relatively minimal foliation with pseudoeffective $K_{\mathcal{F}}$ on a smooth projective surface $X$, and let $K_{\mathcal{F}}=P+N$ be the Zariski decomposition of $K_{\mathcal{F}}$. Assume that there exists a $K_{X}$-negative extremal curve $C \subset X$ with $P \cdot C=0$. Then the Kodaira dimension of $\mathcal{F}$ is either 0 or 1 . Moreover, if $\operatorname{kod}(\mathcal{F})=1$, then the image of $C$ in the canonical model $\pi: X \rightarrow Z$ of $\mathcal{F}$ is proportional to $\pi_{*} K_{\mathcal{F}}$.

Proof. If $C$ is an extremal ray with $C^{2} \geqslant 1$, then the Hodge index theorem implies that $P$ is numerically zero. Theorem 2.18 implies that $\operatorname{kod}(\mathcal{F})=0$.

If instead $C^{2}=0$, then $P$ is numerically equivalent to a non-negative multiple of $C$, and we deduce that either $\nu(\mathcal{F})=0$ or $\nu(\mathcal{F})=1$. The case $\nu(\mathcal{F})=0$ follows as before. If $\nu(\mathcal{F})=1$, since $P$ is numerically equivalent to an effective divisor, we can apply Theorem 2.20 and Lemma 2.21 to deduce that $\operatorname{kod}(\mathcal{F})=1$.

From now on, assume that $C^{2}=-1$, and let $\pi: X \rightarrow Y$ be the contraction of $\mathcal{F}$ into its canonical model. If $C$ is not contracted by $\pi$, then write $\pi^{*} \pi_{*} C=C+\sum a_{i} E_{i}$, where $a_{i}>0$ and the $E_{i}$ are $\pi$-exceptional divisors. Thus, $\pi_{*} P \cdot \pi_{*} C=P \cdot \pi^{*} \pi_{*} C=P \cdot C$ since $P$ is the pull-back of a nef divisor from $Y$ and hence $\pi$-exceptional curves intersect $P$ trivially. As we are assuming that $P \cdot C=0$, we deduce from Hodge index theorem that either $P$ is numerically trivial, or 


\section{EFFECTIVE ALGEBRAIC INTEGRATION IN BOUNDED GENUS}

$\pi_{*} C^{2}=0$ and $\pi_{*} P$ is numerically equivalent to a positive multiple of $\pi_{*} C$. Hence $\nu(\mathcal{F}) \in\{0,1\}$. As before, we obtain that in both cases $\nu(\mathcal{F})=\operatorname{kod}(\mathcal{F})$.

Now, suppose that $C$ is contracted by $\pi$. In this case, $C$ is $\mathcal{F}$-invariant according to Theorem 2.17. Since $C^{2}=-1$ and $\mathcal{F}$ is relatively minimal, we have that $Z(\mathcal{F}, C) \geqslant 3$. Notice that $K_{\mathcal{F}} \cdot C=-2+Z(\mathcal{F}, C)$ and, as we are assuming that $P \cdot C=0$, according to Lemma 2.14 we also have that $K_{\mathcal{F}} \cdot C=\sum_{i=1}^{k} 1 / o_{i}$, where the $o_{i}$ are the orders of the Hirzebruch-Jung strings intersecting $C$. Then we must have that $k=2$ and $o_{1}=o_{2}=2$; or $k=3$ and $\left(o_{1}, o_{2}, o_{3}\right) \in\{(2,3,6),(3,3,3)\}$; or $k=4$ and $\left(o_{1}, o_{2}, o_{3}, o_{4}\right)=(2,2,2,2)$. If we contract the Hirzebruch-Jung strings intersecting $C$, we obtain that the direct image of $C$ has non-negative self-intersection; cf. [McQ08, Remark III.2.2]. Thus $C$ cannot be contracted by $\pi$, contrary to our assumption.

\subsection{Kodaira dimension zero}

Lemma 6.2. Let $\mathcal{F}$ be a relatively minimal foliation with pseudoeffective $K_{\mathcal{F}}$ on a smooth projective surface $X$. If $\pi: X \rightarrow Z$ is the contraction of the negative part of $K_{\mathcal{F}}$ (that is, $\pi_{*} \mathcal{F}$ is a nef model of $\left.\mathcal{F}\right)$ and we write $K_{X}+\Delta=\pi^{*} K_{Z}$, then $\mathrm{i}(\mathcal{F}) N-\Delta$ is effective.

Proof. If $E_{1}, \ldots, E_{k}$ are the exceptional divisors of $\pi$, then $\Delta$ is defined by the relations

$$
\Delta \cdot E_{i}=-K_{X} \cdot E_{i}=2+E_{i}^{2} .
$$

Notice that $2+E_{i}^{2} \leqslant 0$ for every $i$, while $2+E_{i}^{2} \geqslant\left(E_{1}+\cdots+E_{k}\right) \cdot E_{i}$ for every $i$, and the latter inequality is strict when $E_{i}$ is either a handle or a tail in a Hirzebruch-Jung string. Therefore, by [KM98, Corollary 4.2], the coefficients of $\Delta$ lie in $[0,1)$. Since $N$ is effective, the lemma follows.

Proposition 6.3. Let $\mathcal{F}$ be a relatively minimal foliation of Kodaira dimension zero on a smooth projective surface $X$. If $\pi: X \rightarrow Z$ is the contraction of the negative part of the Zariski decomposition of $K_{\mathcal{F}}$ and $(X, \Delta)$ is the pair satisfying $K_{X}+\Delta=\pi^{*} K_{Z}$, then the adjoint dimension and the numerical adjoint dimension of $\mathcal{F}$ coincide with the Kodaira dimension of $(X, \Delta)$. Moreover, if $\operatorname{adj}(\mathcal{F}) \geqslant 0$, then $\operatorname{eff}(\mathcal{F}) \geqslant 1 /(\mathrm{i}(\mathcal{F})+1) \geqslant 1 / 13$.

Proof. Let $K_{\mathcal{F}}=P+N$ be the Zariski decomposition of $K_{\mathcal{F}}$. Since we are assuming that $\mathcal{F}$ has Kodaira dimension zero, we have that $P \sim_{\mathbb{Q}} 0$. Let $\pi: X \rightarrow Z$ be the contraction of the support of $N$, and notice that we can write

$$
K_{\mathcal{F}}+\varepsilon K_{X}=\varepsilon \pi^{*} K_{Z}+(N-\varepsilon \Delta) .
$$

Assume that $\varepsilon$ is rational and satisfies $\varepsilon<1 / \mathrm{i}(\mathcal{F})$. Lemma 6.2 implies that $(N-\varepsilon \Delta)$ is effective. Hence for any $k$ sufficiently divisible, $h^{0}\left(X, k\left(\varepsilon \pi^{*} K_{Z}+(N-\varepsilon \Delta)\right)\right) \geqslant h^{0}\left(X, k \varepsilon \pi^{*} K_{Z}\right)=$ $h^{0}\left(Z, k \varepsilon K_{Z}\right)$. Since every irreducible component $E$ of the support of $(N-\varepsilon \Delta)$ is $\pi$-exceptional, we also have the opposite inequality. This shows that the Kodaira dimension of $Z$ is equal to the adjoint dimension of $\mathcal{F}$.

To verify that the adjoint dimension and the numerical adjoint dimension of $\mathcal{F}$ coincide, first observe that every irreducible component $E$ of $N-\varepsilon \Delta$ satisfies $\pi^{*} K_{Z} \cdot E=0$. Therefore, the numerical dimension of $K_{\mathcal{F}}+\varepsilon K_{X}$ coincides with the numerical dimension of $K_{Z}$. As the numerical dimension of $K_{Z}$ and the Kodaira dimension of $(X, \Delta)$ coincide, the proposition follows.

Finally, the last part of the statement follows from the fact that for a relatively minimal foliation $\mathcal{F}$ of Kodaira dimension zero, $\mathrm{i}(\mathcal{F}) \leqslant 12$; see $(2.1)$. 


\section{J. V. PEREIRA AND R. Svaldi}

\subsection{Kodaira dimension one}

Proposition 6.4. Let $\mathcal{F}$ be a relatively minimal foliation of Kodaira dimension one on a smooth projective surface $X$. Let $g$ be the genus of a general fiber of the Iitaka fibration of $\mathcal{F}$. If $g=0$, then $\operatorname{adj}(\mathcal{F})=\operatorname{adj}_{\text {num }}(\mathcal{F})=-\infty$. Otherwise,

$$
\operatorname{adj}(\mathcal{F})=\operatorname{adj}_{\text {num }}(\mathcal{F})=\min \{g, 2\} \quad \text { and } \quad \operatorname{eff}(\mathcal{F}) \geqslant \frac{1}{4 \mathrm{i}(\mathcal{F})+1} .
$$

Proof. Let $f: X \rightarrow B$ be the Iitaka fibration of $\mathcal{F}$. First, assume that $g=0$. Then for a general fiber $F$ of $f$, we have that $K_{\mathcal{F}} \cdot F=0$ and $K_{X} \cdot F=-2$. Hence $K_{\mathcal{F}}+\varepsilon K_{X}$ is not pseudoeffective for every $\varepsilon>0$. It follows that $\operatorname{adj}(\mathcal{F})=\operatorname{adj}_{\text {num }}(\mathcal{F})=-\infty$.

Now, assume that $g \geqslant 1$. Let $K_{\mathcal{F}}=P+N$ be the Zariski decomposition of $K_{\mathcal{F}}$, and let $\pi: X \rightarrow Z$ be the contraction of the negative part of $K_{\mathcal{F}}$. Denote the direct image of $\mathcal{F}$ by $\mathcal{G}$. We claim that $4 \mathrm{i}(\mathcal{F}) K_{\mathcal{G}}+K_{Z}$ is nef. Suppose that it is not, and let $D$ be an effective divisor such that $\left(4 \mathrm{i}(\mathcal{F}) K_{\mathcal{G}}+K_{Z}\right) \cdot D<0$. By the cone theorem, we can numerically decompose $D$ as a sum $\sum a_{i} C_{i}+R$, where $R$ is a pseudoeffective divisor and satisfies $K_{Z} \cdot R \geqslant 0$, the $C_{i}$ are $K_{Z}$-negative extremal rays satisfying $0<-K_{Z} \cdot C_{i} \leqslant 4$, and $a_{i} \in \mathbb{R}_{>0}$. Therefore, there exists a $K_{Z}$-negative extremal ray $C$ such that $\left(4 \mathrm{i}(\mathcal{F}) K_{\mathcal{G}}+K_{Z}\right) \cdot C<0$. If $K_{\mathcal{G}} \cdot C=0$, then Lemma 6.1 implies that $C$ is numerically proportional to $K_{\mathcal{G}}$. In fact, denoting by $\tilde{C}$ the strict transform of $C$ on $X$, we have that $P \cdot \tilde{C}=\pi^{K_{\mathcal{G}}} \cdot \tilde{C}=0$. Consequently, $C$ is proportional to a general fiber of $f \circ \pi^{-1}$ and must intersect $K_{Z}$ non-negatively. Thus, $K_{\mathcal{G}} \cdot C>0$. Since $\mathrm{i}(F) K_{\mathcal{G}}$ is Cartier, we deduce that $4 \mathrm{i}(\mathcal{F}) K_{\mathcal{G}} \cdot C \geqslant 4$. It follows that also in this case, $\left(4 \mathrm{i}(\mathcal{F}) K_{\mathcal{G}}+K_{Z}\right) \cdot C \geqslant 0$. We conclude that $4 \mathrm{i}(\mathcal{F}) K_{\mathcal{G}}+K_{Z}$ is nef. Consequently, we obtain that

$$
K_{\mathcal{F}}+\frac{1}{4 \mathrm{i}(\mathcal{F})} K_{X}=\pi^{*}\left(K_{\mathcal{G}}+\frac{1}{4 \mathrm{i}(\mathcal{F})} K_{Z}\right)+\left(N-\frac{1}{4 \mathrm{i}(\mathcal{F})} \Delta\right),
$$

where $\Delta$ is defined by $K_{X}+\Delta=\pi^{*} K_{Z}$. Since the singularities of $Z$ are klt, it follows that $N-(1 / 4 \mathrm{i}(\mathcal{F})) \Delta$ is effective and that $K_{\mathcal{F}}+(1 / 4 \mathrm{i}(\mathcal{F})) K_{X}$ is pseudoeffective. Thus, eff $(\mathcal{F}) \geqslant$ $1 /(4 \mathrm{i}(\mathcal{F})+1)$.

It remains to determine the adjoint dimension of $\mathcal{F}$. For that, notice that (6.1) is the Zariski decomposition of $K_{\mathcal{F}}+(1 / 4 \mathrm{i}(\mathcal{F})) K_{X}$. When $g=1$, since $K_{X}$ is trivial when restricted to the general fiber of $f$, it follows that the positive part $\pi^{*}\left(K_{\mathcal{G}}+(1 / 4 \mathrm{i}(\mathcal{F})) K_{Z}\right)$ is numerically proportional to a general fiber and also that there exists an a effective $\mathbb{Q}$-divisor $D$ on $B$ such that $\pi^{*}\left(K_{\mathcal{G}}+(1 / 4 \mathrm{i}(\mathcal{F})) K_{Z}\right)=f^{*} B$. Hence, $\operatorname{adj}_{\text {num }}(\mathcal{F})=\operatorname{adj}(\mathcal{F})=1$.

To prove the claim for $g \geqslant 2$, it suffices to verify that $\pi^{*}\left(K_{\mathcal{G}}+\varepsilon K_{Z}\right)^{2}>0$ for $\varepsilon$ sufficiently small. If this were not the case, then $K_{\mathcal{G}} \cdot K_{Z}=0$ and $K_{Z} \cdot K_{Z}=0$. The Hodge index theorem would imply that $\pi^{*} K_{Z}$ is proportional to a general fiber $f$. But this is not possible since $\pi^{*} K_{Z}$. $F=2 g-2>0$ for any fiber $F$ of $f$.

\subsection{Kodaira dimension two and non-abundant foliations}

LEMMA 6.5. Let $\mathcal{F}$ be a relatively minimal foliation with canonical singularities which is not a fibration by rational curves. Let $K_{\mathcal{F}}=P+N$ be the Zariski decomposition of $K_{\mathcal{F}}$. If $\operatorname{kod}(\mathcal{F}) \notin$ $\{0,1\}$, then $P+(1 / 3 \mathrm{i}(\mathcal{F})) K_{X}$ is nef.

Proof. In order to deduce a contradiction, let $C$ be a curve such that $\left(P+1 / 3 \mathrm{i}(\mathcal{F}) K_{X}\right) \cdot C<0$. As in the proof of Proposition 6.3, we can assume that $C$ is a $K_{X}$-negative extremal curve and therefore $K_{X} \cdot C \in\{-3,-2,-1\}$. By Lemma 6.1 , we have that $P \cdot C>0$. Hence,

$$
-K_{X} \cdot C_{i}>3 \mathrm{i}(\mathcal{F})\left(P \cdot C_{i}\right) \geqslant 3
$$




\section{EFFECTIVE ALGEBRAIC INTEGRATION IN BOUNDED GENUS}

gives the sought contradiction.

Proposition 6.6. Let $\mathcal{F}$ be a relatively minimal foliation with canonical singularities and pseudoeffective canonical bundle. If $\operatorname{kod}(\mathcal{F}) \notin\{0,1\}$, then $\operatorname{adj}_{\text {num }}(\mathcal{F})=\operatorname{adj}(\mathcal{F})=2$.

Proof. Let $K_{\mathcal{F}}=P+N$ be the Zariski decomposition of $K_{\mathcal{F}}$. Since $\operatorname{kod}(\mathcal{F}) \neq 0$, we have that $\nu(\mathcal{F}) \geqslant 1$. Lemma 6.5 implies that $P+\varepsilon K_{X}$ is nef for $0<\varepsilon$ sufficiently small.

Assume to the contrary that $\mathcal{F}$ is not of adjoint general type. Then $\left(P+\varepsilon K_{X}\right)^{2}$ must vanish identically. In fact, if $\left(P+\varepsilon K_{X}\right)^{2}$ does not vanish identically, then for $0<\varepsilon$ sufficiently small, $\left(P+\varepsilon K_{X}\right)^{2}>0$ by the nefness of $P+\varepsilon K_{X}$. This implies that $P+\varepsilon K_{X}$ is big for $0<\varepsilon$ sufficiently small, and the same holds true for $K_{\mathcal{F}}+\varepsilon K_{X}$, as $N \geqslant 0$, giving a contradiction. From this observation, it follows that $P^{2}=P \cdot K_{X}=K_{X}{ }^{2}=0$. Lemma $2.21 \operatorname{implies}$ that $\operatorname{kod}(\mathcal{F}) \geqslant 0$. But this is not possible by the assumptions in the statement of the proposition. Hence, we reach the desired contradiction, and the result follows.

\subsection{Characterization of rational fibrations (Proof of Theorem B)}

One immediate consequence of the classification of foliations according to their adjoint dimension is the characterization of rational fibrations stated in the introduction as Theorem B.

TheOREM 6.7. Let $\mathcal{F}$ be a foliation with canonical singularities on a smooth projective surface $X$. Then $\mathcal{F}$ is a rational fibration if and only if $h^{0}\left(X, K_{\mathcal{F}}{ }^{\otimes m} \otimes N_{\mathcal{F}}^{*}{ }^{\otimes n}\right)=0$ for every $m>0$ and every $n \geqslant 0$.

Proof. If $\operatorname{adj}(\mathcal{F}) \geqslant 0$, then $h^{0}\left(X, K_{\mathcal{F}}{ }^{\otimes m} \otimes N_{\mathcal{F}}^{*}{ }^{\otimes n}\right) \neq 0$ for some $m, n>0$, by definition. If instead $\operatorname{adj}(\mathcal{F})=-\infty$ and $\mathcal{F}$ is not a fibration by rational curves, then by Theorem 2.6, the bundle $K_{\mathcal{F}}$ must pseudoeffective and $\operatorname{kod}(\mathcal{F})=-\infty$. Moreover, we can assume that $\mathcal{F}$ is relatively minimal by Proposition 3.6. But then Proposition 6.6 gives a contradiction, as it implies that $\operatorname{adj}(\mathcal{F})=2$.

For foliations on smooth surfaces of Kodaira dimension zero or one, $h^{0}\left(X, K_{\mathcal{F}}{ }^{\otimes n}\right)>0$ for some $n$ between 1 and 12; see [Per05] and [CLP16, Section 4]. It is a simple matter to obtain the effective non-vanishing of $h^{0}\left(X, K_{\mathcal{F}}^{\otimes n} \otimes N_{\mathcal{F}}^{*} \otimes m\right)$ for foliations $\mathcal{F}$ of adjoint general type as functions of their index $i(\mathcal{F})$. This is what we did in the proof of Proposition 5.1 when $\kappa(\mathcal{F})=2$. The real question here is whether one can do this regardless of the index of the foliation.

Problem 6.8. Find universal bounds on $(n, m) \in \mathbb{Z}_{>0} \times \mathbb{Z}_{>0}$ in order to ensure the non-vanishing of $h^{0}\left(X, K_{\mathcal{F}}^{\otimes m} \otimes N_{\mathcal{F}}^{*} \otimes n\right)$ for foliations of adjoint general type.

For bounded families of foliations, the results of Section 7 imply the existence of bounds depending on the family.

\section{Variation in moduli}

\subsection{Families of foliations}

We start by spelling out the definition of a family of foliated surfaces.

Definition 7.1. Let $\pi: \mathscr{X} \rightarrow T$ be a family of smooth projective surfaces; that is, $\mathscr{X}$ and $T$ are irreducible complex manifolds, and $\pi$ is a proper submersion with projective surfaces as fibers. A family of foliations parametrized by $T$ is a foliation $\mathscr{F}$ of dimension one on $\mathscr{X}$ which is everywhere tangent to the fibers of $\pi$. If $\mathscr{X}, T, \pi$, and $\mathscr{F}$ are all algebraic, then we say that $\mathscr{F}$ is an algebraic family of foliations. 


\section{J. V. PeReira AND R. Svaldi}

To simplify the notation, we will denote the (schematic) fiber of $\pi$ over a point $t \in T$ by $\mathscr{X}_{t}$ and $\mathscr{F}_{\mid} \mathscr{X}_{t}$ by $\mathscr{F}_{t}$.

Notice that in Definition 7.1, we do not impose any conditions on the nature of the singularities of $\mathscr{F}$, contrary to what is done in [Bru01], where the singularities are supposed to be reduced. Also, when the dimension of $T$ is at least two, it may happen that some fibers of $\pi$ are contained in the singular set of $\mathscr{F}$.

Remark 7.2. Although no assumption is made on the nature of the singularities, the singular set of $\mathscr{F}$ has codimension at least two as the singular set of any foliation on a smooth manifold. Then there exists a non-empty Zariski-open subset $U \subset T$ such that $\operatorname{sing}\left(\mathscr{F}_{t}\right)=\operatorname{sing}(\mathscr{F}) \cap \mathscr{X}_{t}$ for every $t \in U$. In particular, $K_{\mathscr{F}_{t}}=\left(K_{\mathscr{F}}\right)_{\mid \mathscr{X}_{t}}$ for every $t \in U$. Note also that for every $t \in U$, we have the equality $\operatorname{det} N_{\mathscr{F} \mid \mathscr{X}_{t}}^{*}=N_{\mathscr{F}_{t}}^{*}$.

It is useful to think of an algebraic family of foliations parametrized by $T$ as a foliation defined over the function field $\mathbb{C}(T)$. Algebraic properties of a very general member $\mathscr{F}_{t}$ of the familylike the existence of invariant algebraic curves, rational first integrals, transversely projective structures - are displayed already when one considers the foliation as defined over $\mathbb{C}(T)$. Also, the Kodaira dimension (respectively, adjoint dimension) of the foliation defined over $\mathbb{C}(T)$ coincides with the Kodaira dimension (respectively, adjoint dimension) of a very general member of the family.

\subsection{Partial reduction of singularities for families}

When the singularities of a family of foliations $\mathcal{F}$ on smooth surfaces are reduced, Brunella [Bru01] showed that the Kodaira dimension of the foliations is constant in the family. Later, Cascini and Floris proved that for families of foliations with reduced singularities and for $m$ sufficiently large, the $m$ th plurigenus $h^{0}\left(\mathcal{X}_{t}, m K_{\mathcal{F} t}\right)$ is constant. They also presented examples of families of foliations with reduced singularities which have non-constant $m$ th plurigenera for small values of $m$; see [CF18, Section 3.4].

When we do not restrict to families of foliations with reduced singularities, one of the sources of difficulties in applying birational techniques similar to those of [CF18] to understand the behavior of the plurigenera comes from the fact that canonical singularities are not stable in the Zariski topology: the set of foliations with at worst canonical singularities can fail to be Zariski open, as the family of foliations on $\mathbb{C}^{2}$ parametrized by $\mathbb{C}$ and defined by $x d y-t y d x$ shows. In this family, the singularity at the origin is canonical if and only if $t \notin \mathbb{Q}_{+}$. Thus, a very general foliation in the family has canonical singularities, but the set of foliations with non-canonical singularities is Zariski dense. This unpleasant situation can be avoided if, instead, one considers $\varepsilon$-canonical singularities for $\varepsilon>0$.

Lemma 7.3. Let $\mathscr{F}$ be an algebraic family of foliations parametrized by an algebraic variety $T$. If $0<\varepsilon<1 / 4$, then the subset of $T$ corresponding to foliations with isolated and $\varepsilon$-canonical singularities is a Zariski-open subset of $T$.

Proof. This is a simple consequence of Corollary 4.10. If a singularity is not $\varepsilon$-canonical with $0<\varepsilon<1 / 4$, then either its linear part is nilpotent, or the singularity is formally equivalent to one of the finitely many singularities of the form $p x \partial / \partial x+q y \partial / \partial y$ with $p$ and $q$ relatively prime positive integers satisfying $\varphi(p, q)<\varepsilon /(1-\varepsilon)$ (see Definition 4.6 for the meaning of $\varphi$ ). Since both conditions are clearly closed, the lemma follows. 


\section{EFFECTIVE ALGEBRAIC INTEGRATION IN BOUNDED GENUS}

Proposition 7.4. Given an algebraic family of foliation $\mathscr{F}$ parametrized by an algebraic variety $T$ and a real number $\varepsilon>0$, there exist a non-empty Zariski-open subset $U \subset T$ and a family of foliations $\mathscr{G}$ on $\mathscr{Y} \rightarrow U$ obtained from $\mathscr{F}_{\mid U}$ by a finite composition of blow-ups over (multi-)sections such that for every closed point $t \in U$, the foliation $\mathscr{G}_{t}$ has at worst $\varepsilon$-canonical singularities.

Proof. First, replace $T$ with a Zariski-open subset in such a way that the singular scheme of $\mathscr{F}$ becomes flat over $T$. In particular, every irreducible component of the singular set of $\mathscr{F}$ is a multi-section of the projection to $T$.

We will say that an irreducible component $\Sigma$ of the singular set of $\mathscr{F}$ is reduced when, for a very general $t \in T$, the points in $\Sigma_{t}$ are reduced singularities for $\mathscr{F}_{t}$.

If we interpret $\mathscr{F}$ as a foliation $\mathscr{F} \overline{\mathbb{C}(T)}$ over $\overline{\mathbb{C}(T)}$, the Zariski closure of the function field of $T$, then the reduced and non-reduced singularities of $\mathscr{F} \overline{\mathbb{C}(T)}$ correspond to irreducible components of $\operatorname{sing}(\mathscr{F})$ having, respectively, reduced and non-reduced singularities at the very general point.

The proof of Seidenberg's theorem presented in [MM80, Appendix 1] and [Bru04] works for arbitrary algebraically closed fields of characteristic zero. It consists in showing that the iterated blow-ups of non-reduced points will eventually lead to foliations with only reduced singularities. First, one aims to obtain foliations with all singularities having non-trivial linear part. To achieve this, one controls the multiplicity of the singularities of the foliation by means of the Van den Essen formula [vdE79, Section 2, Theorem 1-2, pp. 52-53] (see also [MM80, Appendix 1, (2.1) and (2.2)]) for arbitrary algebraically closed fields of characteristic zero. Then to go from nontrivial linear part to non-nilpotent linear part, one carries out explicit algebraic computations; for details, see [MM80, Appendix 1, Theorem 2]. Finally, to go from the non-nilpotent linear part to the quotient of eigenvalues not belonging to $\mathbb{Q}>0$, one relies on the Euclidean division algorithm [MM80, Appendix 1, Theorem 3].

Therefore, blowing up non-reduced multi-sections, restricting $T$ to suitable Zariski-open subsets, and repeating if necessary, will lead to the sought family of foliations over a Zariski-open subset of $T$ with all irreducible components of its singular set reducible. We apply Lemma 7.3 to conclude.

\subsection{Families of foliations of negative adjoint dimension}

Foliations of negative adjoint dimension also behave better in families compared to foliations of negative Kodaira dimension.

Lemma 7.5. Let $(\pi: \mathscr{X} \rightarrow T, \mathscr{F})$ be an algebraic family of foliations. If for a very general closed point $t_{0} \in T$, the foliation $\mathscr{F}_{t_{0}}$ is reduced and has negative adjoint dimension, then there exists a non-empty Zariski-open subset $U \subset T$ such that for every closed point $t \in U$, the foliation $\mathscr{F}_{t}$ has negative adjoint dimension.

Proof. First, assume that for a very general point $t \in T$, the foliation $\mathscr{F}_{t}$ has Kodaira dimension one. Since the adjoint dimension is negative, $\mathscr{F} t$ must be a Riccati foliation. It follows from [CLP16, Proposition 4.3] that for some $n \leqslant 42$, the linear system $\left|K_{\mathscr{F}_{t}}^{\otimes n}\right|$ is non-empty and defines the reference rational fibration. Moreover, the general fiber of the reference fibration intersects $K_{\mathscr{F}}$ trivially. After replacing $T$ with a Zariski-open subset, we can assume that $\operatorname{sing}\left(\mathscr{F}_{t}\right)=\operatorname{sing}(\mathscr{F}) \cap \mathscr{X}_{t}$; see Remark 7.2 . We can also assume that $\pi_{*} K_{\mathscr{F}}^{\otimes n}$ is locally free. Consider the rational map defined by pluricanonical sections

$$
\mathscr{X} \rightarrow \mathbb{P}\left(\left(\pi_{*} K_{\mathscr{F}}^{\otimes n}\right)^{*}\right) \text {. }
$$




\section{J. V. Pereira And R. Svaldi}

We can further restrict $T$ in order to get an actual morphism. Let $P: \mathscr{X} \rightarrow B \subset \mathbb{P}\left(\left(\pi_{*} K_{\mathscr{F}}^{\otimes n}\right)^{*}\right)$ be such a morphism. We do not claim that $P$ has irreducible fibers, but since $\mathscr{F}_{t}$ is a Riccati foliation of Kodaira dimension one for a very general $t$, it follows that very general fibers of $P$ are smooth curves with rational connected components, completely transverse to $\mathscr{F}_{t}$ and intersecting $K_{\mathscr{F}}$ trivially. Hence, using for instance Ehresmann's fibration theorem, we can conclude that the same holds true for fibers of $F$ over a Zariski-open subset $V$ of $B$. Let $U \subset T$ be a Zariski-open subset contained in the image of $V$ under the natural projection $B \rightarrow T$. We can apply [Bru04, Proposition 4.1] to deduce that for every $t \in U$, the foliation $\mathscr{F}_{t}$ is a Riccati foliation and as such has negative adjoint dimension.

Now, assume that for a very general point $t \in T$, the foliation $\mathscr{F}_{t}$ has Kodaira dimension zero. We will make use of the Lefschetz principle to deal with this case. Since the family $\mathscr{F}$ is defined by finitely many equations, we can consider a finitely generated extension $K$ of $\mathbb{Q}$ over which everything in sight is defined. Embed $K$ into $\mathbb{C}$ and apply Theorem 2.18. We deduce that after restricting $T$ to a Zariski-open subset $U$ and base changing the family $\mathscr{F}$ through an étale covering $V \rightarrow U$, we obtain that the resulting family $\mathscr{X}^{\prime} \rightarrow U$ is birationally equivalent to a finite quotient of a smooth family of foliations $\mathscr{G}$ on $\mathscr{Z} \rightarrow V$ defined by global holomorphic vector fields. Since we are assuming that for a very general $t \in T$, the foliation has negative adjoint dimension, it follows that the very general fiber of $\mathscr{Z} \rightarrow V$ is a surface of negative Kodaira dimension and the corresponding foliation is a Riccati foliation. It follows that for every $t \in U$, the foliation $\mathscr{F}_{t}$ has negative adjoint dimension.

Finally, let us assume that for a very general $t \in T$, the foliation $\mathscr{F}_{t}$ is a rational fibration. Let $U \subset T$ be a Zariski-open subset over which every irreducible component of the singular scheme of $\mathscr{F}$ is flat. As we are assuming that $\mathscr{F}_{t}$ is a foliation with reduced singularities tangent to a rational fibration for a very general $t \in T$, it follows that for a very general $t \in U$, the singularities of $\mathscr{F}_{t}$ have an invertible linear part and quotient of eigenvalues in $\mathbb{Q}<0$. The flatness of the singular scheme of $\mathscr{F}$ over $U$ guarantees that the singularities of $\mathscr{F} t$ have an invertible linear part for every $t \in U$. The continuity of the quotient of eigenvalues ensures that they are constant functions of $t \in U$. Thus, for every $t \in U$, the foliations $\mathscr{F}_{t}$ have reduced singularities. It follows from [Bru01, Lemma 1] that $\mathscr{F}_{t}$ is a rational fibration for every $t \in U$.

\subsection{Boundedness of the effective threshold in families}

We now have all the ingredients to prove the result mentioned at the end of Section 4.2.

Theorem 7.6. Let $(\pi: \mathscr{X} \rightarrow T, \mathscr{F})$ be an algebraic family of foliations. Then there exists a $\delta>0$ such that for every $t \in T$, the following holds true: $\operatorname{adj}\left(\mathscr{F}_{t}\right)=-\infty$ or $\operatorname{eff}\left(\mathscr{F}_{t}\right) \geqslant \delta$. In other words, if $\operatorname{eff}\left(\mathscr{F}_{t}\right)<\delta$, then $\operatorname{adj}\left(\mathscr{F}_{t}\right)=-\infty$.

Proof. The proof is by Noetherian induction. Therefore, it suffices to show that the result is valid over a Zariski-open subset of $T$. In particular, after replacing $T$ with a Zariski-open subset, we can assume that $\operatorname{sing}\left(\mathscr{F}_{t}\right)=\operatorname{sing}(\mathscr{F}) \cap \mathscr{X}_{t}$ for every $t \in T$. Proposition 7.4 guarantees that there is no loss of generality in assuming that $\mathscr{F}_{t}$ has canonical singularities for a very general $t \in T$.

If $\operatorname{adj}\left(\mathscr{F}_{t}\right) \geqslant 0$ for a very general $t \in T$, then there exist $m, n>0$ such that $h^{0}\left(\mathscr{X}_{t}, K_{\mathscr{F} t}{ }^{\otimes m} \otimes\right.$ $\left.N_{\mathscr{F} t}^{*}{ }^{\otimes n}\right)>0$ for a very general $t \in T$. Choose $\varepsilon>0$ small enough and apply Proposition 7.4 to obtain a Zariski-open $U \subset T$ such that $\mathscr{F}_{t}$ has at worst $\varepsilon$-canonical singularities for every $t \in U$. Remark 7.2 guarantees that $K_{\mathscr{F} t}^{\otimes m} \otimes N_{\mathscr{F} t}^{*}$ in the restriction of a line bundle on $\mathscr{X}$ to $\pi^{-1}(t)$. By semi-continuity, it follows that $\operatorname{eff}\left(\mathscr{F}_{t}\right) \geqslant n / m$ for every $t \in U$.

If instead $\operatorname{adj}\left(\mathscr{F}_{t}\right)=-\infty$ for a very general $t \in T$, then Lemma 7.5 implies that the same 


\section{EFFECTIVE ALGEBRAIC INTEGRATION IN BOUNDED GENUS}

holds true for every $t$ in a Zariski-open subset of $T$. In any case, we have just proved that the result is true for the restriction of $\mathscr{F}$ to a non-empty Zariski-open subset $U$ of $T$.

The proof continues by passing to irreducible components of $T \backslash U$. To be more precise, let $S$ be a resolution of singularities of one such irreducible component, and let $\mathscr{Y} \rightarrow S$ be the base change of $\mathscr{X} \rightarrow T$ to $S \rightarrow T \backslash U \subset T$. If $v \in H^{0}\left(\mathscr{X}, T_{\mathscr{X} / T} \otimes K_{\mathscr{F}}\right)$ is a twisted vector field defining $\mathscr{F}$, then it induces an element $w \in H^{0}\left(\mathscr{Y}, T_{\mathscr{Y} / S} \otimes\left(K_{\mathscr{F}}\right) \mathscr{Y}\right)$. If $w$ is identically zero, then the points of $S$ do not correspond to foliations and we do nothing. Otherwise, we divide $w$ by the divisorial components of its singular set in order to obtain the defining twisted vector field of a family of foliations over $S$. Since we are back to our original problem, but with a lower-dimensional base, we are done by Noetherian induction.

\section{Foliations with rational first integrals}

\subsection{Transversely affine and transversely projective foliations}

A foliation on a projective surface $X$ is called transversely affine if for any rational 1-form $\omega_{0}$ defining $\mathcal{F}$, there exists a rational 1 -form $\omega_{1}$ such that

$$
d \omega_{0}=\omega_{0} \wedge \omega_{1} \quad \text { and } \quad d \omega_{1}=0 .
$$

Similarly, a foliation $\mathcal{F}$ on $X$ is called transversely projective if for any rational 1-form $\omega_{0}$ defining $\mathcal{F}$, there exist rational 1 -forms $\omega_{1}$ and $\omega_{2}$ such that

$$
d \omega_{0}=\omega_{0} \wedge \omega_{1}, \quad d \omega_{1}=2 \omega_{0} \wedge \omega_{2}, \quad \text { and } \quad d \omega_{2}=\omega_{1} \wedge \omega_{2} .
$$

For a thorough discussion about transversely affine and transversely projective foliations of codimension one on projective manifolds, the reader should consult [CP14] and [LPT16], respectively.

\subsection{Statement of the main result}

This section is devoted to the proof of the following result.

Theorem 8.1. Let $(\pi: \mathscr{X} \rightarrow T, \mathscr{F})$ be an algebraic family of foliations and $g \geqslant 0$ be an integer. Let $\Sigma_{g} \subset T$ be the Zariski closure of the set of parameters corresponding to foliations birationally equivalent to a fibration of geometric genus at most $g$. Then for every $t \in \Sigma_{g}$, the foliation $\mathscr{F}_{t}$ is transversely projective.

If one considers the universal family of degree $d$ foliations on $\mathbb{P}^{2}$, then one promptly realizes that Theorem $\mathrm{C}$ is nothing but a particular case of this more general statement.

\subsection{Example}

Before dealing with the proof of Theorem 8.1, let us analyze the Zariski closure of the set of foliations admitting a rational first integral in a family derived from the Gauss hypergeometric equation.

Whenever $c \notin \mathbb{Z}$, the Gauss hypergeometric equation

$$
z(1-z) w^{\prime \prime}+(c-(a+b+1) z) w^{\prime}-a b w=0
$$

admits as general solution in a neighborhood of the origin the function

$$
\varphi(z)=C_{1} F(a, b, c ; z)+C_{2} z^{1-c} F(a-c+1, b-c+1,2-c ; z),
$$




\section{J. V. Pereira And R. Svaldi}

where $C_{1}$ and $C_{2}$ are arbitrary constants to be determined by boundary conditions and

$$
F(a, b, c ; z)=1+\sum \frac{(a)_{n}(b)_{n}}{(c)_{n}} z^{n}, \quad(p)_{n}:=p(p+1)(p+2) \cdots(p+n-1) .
$$

The change of variable $y(z)=-d \log w(z)$ associates a Riccati equation/foliation with any second-order differential equation. In this new coordinate, the family of foliations induced by the Gauss hypergeometric equation can be written as

$$
\omega=z(1-z) d y-z(1-z) y^{2}+(c-(a+b+1) z) y+a b d z .
$$

If $\varphi(z)$ is an arbitrary solution of the Gauss hypergeometric equation, then $y=-d \log \varphi(z)$ is a solution of the corresponding Riccati equation. If we choose $c \in \mathbb{Q}-\mathbb{Z}, a \in \mathbb{Z}_{<0}$, and $b=c-1+\beta$, where $\beta \in \mathbb{Z}_{<0}$, then it is clear from the explicit form of the solutions that all the leaves of the foliation corresponding to this choice of parameters are algebraic. It follows that the set of foliations in this family admitting a rational integral is Zariski dense.

On the one hand, for a very generic choice of parameters, the monodromy group of the Gauss hypergeometric equation is Zariski dense in Aut $\left(\mathbb{P}^{1}\right)$; see for instance [Yos97, Chapter 4, Section 5]. On the other hand, in the case of transversely affine Riccati foliations, the monodromy group is not Zariski dense (it must be a solvable subgroup of Aut $\left(\mathbb{P}^{1}\right)$. Therefore, for a very generic choice of parameters, the foliation defined by $\omega$ is transversely projective but not transversely affine.

To conclude, we point out that for the choice of parameters made above, the foliations are birationally equivalent to fibrations by rational curves. Hence, one cannot hope to replace transversely projective with transversely affine in the statement of Theorem 8.1.

\subsection{Non-isotrivial fibrations}

We now start the proof of Theorem 8.1. We first treat the case of foliations birationally equivalent to non-isotrivial fibrations.

Proposition 8.2. Let $g \geqslant 1$ be a natural number, and let $(\pi: \mathscr{X} \rightarrow T, \mathscr{F})$ be an algebraic family of foliations. The Zariski closure in $T$ of the set of parameters corresponding to foliations birationally equivalent to non-isotrivial fibrations of genus at most $g$ consists of foliations admitting rational first integrals.

Proof. According to [CP06, Proposition 2.1], it suffices to prove that the fibers of the nonisotrivial fibrations in the family belong to a bounded family of curves.

For $g=1$, the boundedness is clear since the fibers of a non-isotrivial elliptic fibration $\mathscr{F}_{t}$

are contained in zero sets of sections of $K_{\mathscr{F}}{ }_{t}^{\otimes 12}$; see for instance [CLP16, Proposition 4.2]. The boundedness of fibers of non-isotrivial fibrations of genus $g \geqslant 2$ is guaranteed by Theorem A.

\subsection{Isotrivial fibrations of adjoint general type}

For isotrivial fibrations of adjoint general type, the situation is better when compared to nonisotrivial fibrations as there is no need to bound the genus in order to obtain the boundedness of the leaves.

Proposition 8.3. Let $(\pi: \mathscr{X} \rightarrow T, \mathscr{F})$ be an algebraic family of foliations. The Zariski closure in $T$ of the set of parameters corresponding to foliations of adjoint general type birationally equivalent to isotrivial fibrations consists of foliations admitting rational first integrals. 


\section{EFFECTIVE ALGEBRAIC INTEGRATION IN BOUNDED GENUS}

Proof. If $\mathcal{F}$ is an isotrivial fibration of adjoint general type on a projective surface $X$, then $\mathcal{F}$ has Kodaira dimension one and the Iitaka fibration of $K_{\mathcal{F}}$ is an isotrivial fibration of genus $g \geqslant 2$. According to [CLP16, Proposition 4.10], there are at least two linearly independent sections $\sigma_{1}$ and $\sigma_{2}$ of $K_{\mathcal{F}}{ }^{\otimes k}$ for some $k \leqslant 42$. Consider the rational map $f=\left(\sigma_{1}: \sigma_{2}\right): X \rightarrow \mathbb{P}^{1}$ defined by them. The foliation $\mathcal{G}$ defined by $f$ coincides with the foliation defined by the Iitaka fibration of $K_{\mathcal{F}}$. Its normal bundle is of the form $N_{\mathcal{G}}=f^{*} T_{\mathbb{P}^{1}} \otimes \mathcal{O}_{X}(-\Delta)=K_{\mathcal{F}}{ }^{\otimes 2 k} \otimes \mathcal{O}_{X}(-\Delta)$, where $\Delta$ is an effective divisor. Since the leaves of $\mathcal{F}$ are contained in fibers of the Iitaka fibration of $K_{\mathcal{G}}$, we repeat the argument to obtain the existence of a $k^{\prime} \leqslant 42$ such that the leaves of $\mathcal{F}$ are contained in the zero set of sections of $K_{X} \otimes k^{\prime} \otimes K_{\mathcal{F}} \otimes 2 k^{\prime} k \otimes \mathcal{O}_{X}\left(-k^{\prime} \Delta\right)$. This suffices to prove the boundedness of the leaves of foliations in a family having adjoint general type and birationally equivalent to isotrivial fibrations.

\subsection{First integrals and transverse structures}

Proposition 8.4. Let $\mathcal{F}$ be a foliation on a projective surface $X$. If $\operatorname{adj}(\mathcal{F})<2$, then $\mathcal{F}$ is a transversely projective foliation. Moreover, if $\operatorname{adj}(\mathcal{F}) \in\{0,1\}$, then $\mathcal{F}$ is a transversely affine foliation.

Proof. This is a straightforward consequence of the classification. If $\mathcal{F}$ has adjoint dimension zero, then it is birationally equivalent to a finite quotient of a foliation defined by a closed rational 1-form. Since the property of being transversely affine is invariant by dominant rational maps, $\mathcal{F}$ is transversely affine. If $\mathcal{F}$ has adjoint dimension one, then either $\mathcal{F}$ is a fibration (and therefore clearly transversely affine), or $\mathcal{F}$ is a turbulent foliation, which is well known to be transversely affine (see for instance [Per03, Proposition 22]). Finally, if $\mathcal{F}$ has negative adjoint dimension, then it is a fibration, a Riccati foliation, or a finite quotient of a Riccati foliation. In any case, $\mathcal{F}$ is a transversely projective foliation.

Proposition 8.5. Let $(\pi: \mathscr{X} \rightarrow T, \mathscr{F})$ be an algebraic family of foliations. If for a very general closed point $t_{0} \in T$, the foliation $\mathscr{F}_{t_{0}}$ is a transversely projective foliation, then for every closed point $t \in T$, the foliation $\mathscr{F}_{t}$ is a transversely projective foliation. Similarly, if for a very general closed point $t_{0} \in T$, the foliation $\mathscr{F}_{t_{0}}$ is a transversely affine foliation, then for every closed point $t \in T$, the foliation $\mathscr{F}_{t}$ is a transversely affine foliation.

Proof. We can interpret the family of foliation as a single foliation defined over the function field $\mathbb{C}(T)$. By assumption, this foliation is transversely projective. Hence, there exists a triplet $\left(\omega_{0}, \omega_{1}, \omega_{2}\right)$ of rational differential 1 -forms with coefficients in $\overline{\mathbb{C}(T)}$, the algebraic closure of $\mathbb{C}(T)$, satisfying the equations

$$
d \omega_{0}=\omega_{0} \wedge \omega_{1}, \quad d \omega_{1}=2 \omega_{0} \wedge \omega_{2}, \quad \text { and } \quad d \omega_{2}=\omega_{1} \wedge \omega_{2}
$$

and such that $\omega_{0}$ is a differential 1 -form defined over $\mathbb{C}(T)$ which defines $\mathscr{F}$. According to [Cas02, Lemma 3.2], we can assume that $\omega_{1}$ and $\omega_{2}$ are also defined over $\mathbb{C}(T)$ (there is no need to pass to the algebraic closure). Therefore, over $\mathbb{C}$, we have the equations

$$
d \omega_{0} \wedge d \pi=\omega_{0} \wedge \omega_{1} \wedge d \pi, \quad d \omega_{1} \wedge d \pi=2 \omega_{0} \wedge \omega_{2} \wedge d \pi, \quad \text { and } \quad d \omega_{2} \wedge d \pi=\omega_{1} \wedge \omega_{2} \wedge d \pi .
$$

If $t \in T$ is such that $\pi^{-1}(t)$ is contained neither in the polar set of $\left(\omega_{i}\right)_{\infty}$ for $i=0,1,2$ nor in the zero set of $\omega_{0}$, then the restriction of the triplet $\left(\omega_{0}, \omega_{1}, \omega_{2}\right)$ to the fiber over $t$ defines a (singular) projective structure for the foliation $\mathscr{F}_{t}$ on $X_{t}=\pi^{-1}(t)$.

Let us fix $t_{0} \in T$ such that $X_{0}=\pi^{-1}\left(t_{0}\right)$ is contained in the polar set of $\omega_{i}(i=0,1,2)$ or in the zero set of $\omega_{0}$, and let $f \in \pi^{*} \mathcal{O}_{T, t_{0}}$ be a rational function on $X_{0}$ corresponding to 


\section{J. V. Pereira And R. Svaldi}

a generator of the maximal ideal of $\mathcal{O}_{T, t_{0}}$. Notice that we can replace the triplet $\left(\omega_{0}, \omega_{1}, \omega_{2}\right)$ by $\left(f^{k} \omega_{0}, \omega_{1}, f^{-k} \omega_{2}\right)$. Thus, there is no loss of generality in assuming that $\pi^{-1}\left(t_{0}\right)$ is not contained in $\left(\omega_{0}\right)_{\infty} \cup\left(\omega_{0}\right)_{0}$.

For $i=0,1,2$, let $a_{i}$ be the order of $\omega_{i}$ along $X_{0}$, and set $\alpha_{i}=\operatorname{Res}_{X_{0}} f^{-a_{i}} \omega_{i} \wedge(d f / f)$. As mentioned above, we will assume that $a_{0}=0$ and, therefore, that $\alpha_{0}$ is just the restriction of $\omega_{0}$ to the fiber $X_{0}$.

If $a_{1}$ is negative, then, comparing the orders along $X_{0}$ of $d \omega_{0} \wedge d f$ and of $\omega_{0} \wedge \omega_{1} \wedge d f$, we deduce that $\alpha_{0} \wedge \alpha_{1}=0$, and we can write $\alpha_{0}=g \alpha_{1}$ for some rational function $g \in \mathbb{C}\left(X_{0}\right)$. Let $G \in \mathbb{C}(\mathscr{X})$ be a rational function on $\mathscr{X}$ extending $g$. According to [CLLPT07, (14)], we can replace the triplet $\left(\omega_{0}, \omega_{1}, \omega_{2}\right)$ with the triplet

$$
\left(\omega_{0}, \omega_{1}-f^{-a_{1}} G \omega_{0}, \omega_{2}+f^{-a_{1}} G \omega_{1}+f^{-2 a_{1}} G^{2} \omega_{0}-f^{-a_{1}} d G\right) .
$$

This increases $a_{1}$. After a finite number of changes, we may assume that $a_{0}=0$ and $a_{1} \geqslant 0$.

Finally, if $a_{2}$ is negative and $a_{1}>0$, then $\alpha_{0}$ is closed and it is clear that $\mathscr{F}_{t_{0}}$ is transversely projective. If instead $a_{2}<0$ and $a_{0}=a_{1}=0$, then comparing the orders along $X_{0}$ of $d \omega_{1} \wedge d f$ and $\omega_{0} \wedge \omega_{2} \wedge d f$, we deduce that $\alpha_{0} \wedge \alpha_{2}=0$. Thus, we can write $\alpha_{2}=h \alpha_{0}$ for a suitable rational function $h \in \mathbb{C}\left(X_{0}\right)$. From the equation $d \omega_{2} \wedge d f=\omega_{1} \wedge \omega_{2} \wedge d f$, we deduce that $d \alpha_{2}=\alpha_{1} \wedge \alpha_{2}$. Combining these two identities, we obtain

$$
d\left(h \alpha_{0}\right)=\alpha_{1} \wedge\left(h \alpha_{0}\right) \Longrightarrow d \alpha_{0}=\left(\alpha_{1}-\frac{d h}{h}\right) \wedge \alpha_{0}
$$

Finally, comparing this identity with $d \alpha_{0}=\alpha_{0} \wedge \alpha_{1}$ (first equation), we obtain that $d \alpha_{0}=$ $-\frac{1}{2}(d h / h) \wedge \alpha_{0}$. Thus, $\mathscr{F}_{t_{0}}$ is also transversely projective in this case.

\subsection{Proof of Theorem 8.1 (and of Theorem C)}

Let $(\pi: \mathscr{X} \rightarrow T, \mathscr{F})$ be an algebraic family of foliations and $g \geqslant 0$ be an integer. We want to prove that the Zariski closure of $\Sigma_{g} \subset T$ (subset parametrizing foliations with rational first integral of genus at most $g$ ) corresponds to transversely projective foliations.

If a very general member of the family, say $\mathcal{F}_{t}$, is not of adjoint general type, then Proposition 8.4 implies that $\mathcal{F}_{t}$ is transversely projective. We can apply Proposition 8.5 to conclude that every foliation in the family is also transversely projective. If, instead, a very general member is of adjoint general type, then we will argue as in the proof of Theorem 7.6 to obtain a non-empty Zariski-open subset of $T$ such that every foliation parametrized by this subset is of adjoint general type.

Proposition 7.4 allows us to assume the existence of a non-empty Zariski-open subset $U_{0} \subset T$ such that for a very general $t \in U_{0}$, the foliation $\mathscr{F}_{t}$ has canonical singularities. Since $\mathbb{C}$ is uncountable, we also know that there exist $n, m>0$ and an open subset $U_{1} \subset T$ such that for every $t \in U_{1}$, the linear system $\left|K_{\mathscr{F} t}{ }^{\otimes m} \otimes N_{\mathscr{F} t}^{*}{ }^{\otimes n}\right|$ defines a rational map with two-dimensional image. Notice that there may exist foliations in $U_{0} \cap U_{1}$ which are not of adjoint general type because of the presence of non-canonical singularities. To remedy this, we take $\varepsilon>0$ sufficiently small in order to obtain from Lemma 7.3 a non-empty Zariski-open $U_{2} \subset T$ such that $\mathscr{F}_{t}$ has $\varepsilon$-canonical singularities for every $t \in U_{2}$. Every foliation parametrized by the non-empty Zariski-open set $U=U_{0} \cap U_{1} \cap U_{2}$ is of adjoint general type.

Propositions 8.2 and 8.3 imply that the Zariski closure in $T$ of $\Sigma_{g} \cap U$ corresponds to foliations with rational first integrals. The theorem follows by Noetherian induction. 


\section{EFFECTIVE ALGEBRAIC INTEGRATION IN BOUNDED GENUS}

\section{ACKNOWLEDGEMEnTS}

This collaboration initiated while both authors where visiting James McKernan at UCSD and continued during a visit of the second author to IMPA. We are grateful to both institutions for the favorable working conditions.

The authors wish to thank Calum Spicer for many valuable conversations, Carlo Gasbarri for suggesting an improvement of the bounds in Theorem A, and the anonymous referees for suggesting many improvements in the presentation of this work.

\section{REFERENCES}

BD79 F. Baldassarri and B. Dwork, On second order linear differential equations with algebraic solutions, Amer. J. Math. 101 (1979), no. 1, 42-76; doi:10.2307/2373938.

Bea96 A. Beauville, Complex algebraic surfaces, 2nd edn, London Math. Soc. Stud. Texts, vol. 34 (Cambridge Univ. Press, Cambridge, 1996); doi:10.1017/CB09780511623936.

Bru99 M. Brunella, Minimal models of foliated algebraic surfaces, Bull. Soc. Math. France 127 (1999), no. 2, 289-305; doi:10.24033/bsmf.2349.

Bru01_ Invariance par déformations de la dimension de Kodaira d'un feuilletage sur une surface, in Essays on Geometry and Related Topics, Vols. 1, 2, Monogr. Enseign. Math., vol. 38 (Enseignement Math., Geneva, 2001), 113-132.

Bru04 , Birational geometry of foliations, Publ. Mat. IMPA (Inst. Nac. Mat. Pura Apl., Rio de Janeiro, 2004).

Car94 M. M. Carnicer, The Poincaré problem in the nondicritical case, Ann. of Math. 140 (1994), no. 2, 289-294; doi:10.2307/2118601.

Cas02 G. Casale, Suites de Godbillon-Vey et intégrales premières, C. R. Math. Acad. Sci. Paris 335 (2002), no. 12, 1003-1006; doi:10.1016/S1631-073X (02) 02619-5.

CC97 A. Campillo and M. M. Carnicer, Proximity inequalities and bounds for the degree of invariant curves by foliations of $\mathbb{P}_{\mathbb{C}}^{2}$, Trans. Amer. Math. Soc. 349 (1997), no. 6, 2211-2228; doi: 10.1090/S0002-9947-97-01898-9.

CF18 P. Cascini and E. Floris, On invariance of plurigenera for foliations on surfaces, J. reine angew. Math. 744 (2018), 201-236; doi:10.1515/crelle-2015-0112.

CL91 D. Cerveau and A. Lins Neto, Holomorphic foliations in $\mathbb{C P}(2)$ having an invariant algebraic curve, Ann. Inst. Fourier (Grenoble) 41 (1991), no. 4, 883-903; doi:10.5802/aif.1278.

CLLPT07 D. Cerveau, A. Lins Neto, F. Loray, J. V. Pereira, and F. Touzet, Complex codimension one singular foliations and Godbillon-Vey sequences, Mosc. Math. J. 7 (2007), no. 1, 21-54.

CLP16 G. Cousin, A. Lins Neto, and J. V. Pereira, Toward effective Liouvillian integration, arXiv: 1604.05276.

CP14 G. Cousin and J. V. Pereira, Transversely affine foliations on projective manifolds, Math. Res. Lett. 21 (2014), no. 5, 985-1014; doi:10.4310/MRL.2014.v21.n5.a5.

CP06 S. C. Coutinho and J. V. Pereira, On the density of algebraic foliations without algebraic invariant sets, J. reine angew. Math. 594 (2006), 117-135; doi:10.1515/CRELLE.2006.037.

EK03 E. Esteves and S. Kleiman, Bounds on leaves of one-dimensional foliations, Bull. Braz. Math. Soc. (N.S.) 34 (2003), no. 1, 145-169; doi:10.1007/s00574-003-0006-3.

vdE79 A. van den Essen, Reduction of singularities of the differential equation $A d y=B d x$, Équations différentielles et systèmes de Pfaff dans le champ complexe (Sem., Inst. Rech. Math. Avancée, Strasbourg, 1975), Lecture Notes in Math., vol. 712 (Springer, Berlin, 1979), 44-59; doi: 10.1007/BFb0062814. 


\section{J. V. PEREIRA AND R. Svaldi}

Gra08 J.J. Gray, Linear differential equations and group theory from Riemann to Poincaré, 2nd edn, Mod. Birkhäuser Class. (Birkhäuser Boston, Inc., Boston, MA, 2008); doi:10.1007/ 978-0-8176-4773-5.

GZ97 A. García Zamora, Foliations in algebraic surfaces having a rational first integral, Publ. Mat. 41 (1997), no. 2, 357-373; doi :10.5565/PUBLMAT_41297_03.

Har71 G.H. Hardy, The integration of functions of a single variable, 2nd edn, Cambridge Tracts in Math. and Mathematical Physics, vol. 2 (1916), reprint: Hafner Publishing Co., New York, 1971.

Kle56 F. Klein, Lectures on the icosahedron and the solution of equations of the fifth degree, Dover Publications, Inc., New York, N.Y., 1956.

KM98 J. Kollár and S. Mori, Birational geometry of algebraic varieties, Cambridge Tracts in Math., vol. 134 (Cambridge Univ. Press, Cambridge, 1998); doi:10.1017/CB09780511662560.

Lin02 A. Lins Neto, Some examples for the Poincaré and Painlevé problems, Ann. Sci. École Norm. Sup. (4) 35 (2002), no. 2, 231-266; doi:10.1016/S0012-9593(02)01089-3.

LPT16 F. Loray, J.V. Pereira, and F. Touzet, Representations of quasi-projective groups, flat connections and transversely projective foliations, J. Éc. Polytech. Math. 3 (2016), 263-308; doi:10.5802/jep.34.

McQ08 M. McQuillan, Canonical models of foliations, Pure Appl. Math. Q. 4 (2008), no. 3, part 2, 877-1012; doi:10.4310/PAMQ.2008.v4.n3.a9.

McQ17 Curves on surfaces of mixed characteristic, Eur. J. Math. 3 (2017), no. 3, 433-470; doi:10.1007/s40879-017-0141-9.

Men00 L. G. Mendes, Kodaira dimension of holomorphic singular foliations, Bol. Soc. Brasil. Mat. (N.S.) 31 (2000), no. 2, 127-143; doi:10.1007/BF01244239.

MM80 J.-F. Mattei and R. Moussu, Holonomie et intégrales premières, Ann. Sci. École Norm. Sup. (4) 13 (1980), no. 4, 469-523; doi:10.24033/asens. 1393.

Pai97 P. Painlevé, Leçons sur la théorie analytique des équations différentielles professées à Stockholm (septembre, octobre, novembre 1895) sur l'invitation de S.M. le Roi de Suède et de Norwège (A. Hermann, Paris, 1897).

https://archive.org/details/leonssurlathorie00pain/

Per02 J.V. Pereira, On the Poincaré problem for foliations of general type, Math. Ann. 323 (2002), no. 2, 217-226; doi:10.1007/s002080100277.

Per03 Integrabilidade de folheações holomorfas, Publ. Mat. IMPA (Inst. Nac. Mat. Pura Apl. (IMPA), Rio de Janeiro, 2003).

Per05_ On the height of foliated surfaces with vanishing Kodaira dimension, Publ. Mat. 49 (2005), no. 2, 363-373; doi:10.5565/PUBLMAT_49205_06.

Poi91 H. Poincaré, Sur l'intégration algébrique des équations différentielles du premier ordre et du premier degré, Rend. Circ. Mat. Palermo 5 (1891), no. 1, 161-191; doi:10.1007/BF03015693.

Poi97_ Sur l'intégration algébrique des équations différentielles du premier ordre et du premier degré, Rend. Circ. Mat. Palermo 11 (1897), no. 1, 193-239; doi:10.1007/BF03015916.

Pro03 Yu. G. Prokhorov, On the Zariski decomposition problem, Proc. Steklov Inst. Math. 240 (2003), $37-65$.

vdPS03 M. van der Put and M.F. Singer, Galois theory of linear differential equations, Grundlehren math. Wiss., vol. 328, (Springer-Verlag, Berlin, 2003); doi:10.1007/978-3-642-55750-7.

Rei88 I. Reider, Vector bundles of rank 2 and linear systems on algebraic surfaces, Ann. of Math. 127 (1988), no. 2, 309-316; doi:10.2307/2007055.

Ris70 R.H. Risch, The solution of the problem of integration in finite terms, Bull. Amer. Math. Soc. 76 (1970), no. 3, 605-608; doi:10.1090/S0002-9904-1970-12454-5. 


\section{EFFECTIVE ALGEBRAIC INTEGRATION IN BOUNDED GENUS}

Sch73 H. A. Schwarz, Ueber diejenigen Fälle, in welchen die Gaussische hypergeometrische Reihe eine algebraische Function ihres vierten Elementes darstellt, J. reine angew. Math. 75 (1873), 292-335; doi:10.1515/crll.1873.75.292.

Ser92 F. Serrano, Fibred surfaces and moduli, Duke Math. J. 67 (1992), no. 2, 407-421; doi:10. 1215/S0012-7094-92-06716-0.

Tou16 F. Touzet, On the structure of codimension 1 foliations with pseudoeffective conormal bundle, Foliation Theory in Algebraic Geometry, Simons Symp. (Springer, Cham, 2016), 157-216; doi:10.1007/978-3-319-24460-0_7.

Yos97 M. Yoshida, Hypergeometric functions, my love. Modular interpretations of configuration spaces, Aspects Math., vol. E32 (Friedr. Vieweg \& Sohn, Braunschweig, 1997); doi:10.1007/ 978-3-322-90166-8.

Jorge Vitório Pereira jvp@impa.br

IMPA, Estrada Dona Castorina, 110, Horto, Rio de Janeiro, Brasil

Roberto Svaldi RSvaldi@dpmms.cam.ac.uk

DPMMS, Centre for Mathematical Sciences, University of Cambridge, Wilberforce Road, Cambridge, CB3 0WB, United Kingdom 